\title{
Meta-analysis: Ventilation Strategies and Outcomes of the Acute Respiratory Distress Syndrome and Acute Lung Injury
}

\author{
Christian Putensen, MD, PhD; Nils Theuerkauf, MD; Jörg Zinserling, PhD; Hermann Wrigge, MD, PhD; and Paolo Pelosi, MD
}

Background: Trials have provided conflicting results regarding the effect of different ventilatory strategies on the outcomes of patients with the acute respiratory distress syndrome (ARDS) and acute lung injury.

Purpose: To determine whether ventilation with low tidal volume $(\mathrm{V} T)$ and limited airway pressure or higher positive end-expiratory pressure (PEEP) improves outcomes for patients with ARDS or acute lung injury.

Data Sources: Multiple computerized databases (through March 2009), reference lists of identified articles, and queries of principal investigators. No language restrictions were applied.

Study Selection: Randomized, controlled trials (RCTs) reporting mortality and comparing lower versus higher $\mathrm{V}_{\mathrm{T}}$ ventilation, lower versus higher PEEP, or a combination of both in adults with ARDS or acute lung injury.

Data Extraction: Using a standard protocol, 2 reviewer teams assessed trial eligibility and abstracted data on quality of study design and conduct, population characteristics, intervention, co-interventions, and confounding variables.

Data Synthesis: 4 RCTs tested lower versus higher VT ventilation at similar PEEP in 1149 patients, 3 RCTs compared lower versus higher PEEP at low VT ventilation in 2299 patients, and 2 RCTs compared a combination of higher $\mathrm{V}_{T}$ and lower PEEP ventilation versus lower $\mathrm{V}_{\mathrm{T}}$ and higher PEEP ventilation in 148 patients. Lower $V_{T}$ ventilation reduced hospital mortality (odds ratio, $0.75[95 \% \mathrm{Cl}$, 0.58 to 0.96 ]; $P=0.02$ ) compared with higher $V_{T}$ ventilation at similar PEEP. Higher PEEP did not reduce hospital mortality (odds ratio, $0.86[\mathrm{Cl}, 0.72$ to 1.02$] ; P=0.08$ ) compared with lower PEEP using low $V_{T}$ ventilation. Higher PEEP reduced the need for rescue therapy to prevent life-threatening hypoxemia (odds ratio, $0.51[\mathrm{Cl}$, 0.36 to 0.71$] ; P<0.001$ ) and death (odds ratio, $0.51[\mathrm{Cl}, 0.36$ to 0.71]; $P<0.001$ ) in patients receiving rescue therapies.

Limitations: Pooling according to similar ventilatory strategies resulted in few RCTs analyzed in each group. The benefit of low $\mathrm{V}_{T}$ is derived from only 1 study.

Conclusion: Available evidence from a limited number of RCTs shows better outcomes with routine use of low $\mathrm{V}_{\mathrm{T}}$ but not high PEEP ventilation in unselected patients with ARDS or acute lung injury. High PEEP may help to prevent life-threatening hypoxemia in selected patients.

Primary Funding Source: None.

Ann Intern Med. 2009;151:566-576.

For author affiliations, see end of text.
$\mathrm{T}$ he acute respiratory distress syndrome (ARDS) is clinically characterized by sudden onset, severe hypoxemia, radiographic evidence of bilateral pulmonary infiltration, and absence of left heart failure (1-3). Acute lung injury is a subset of ARDS with less severe impairment in oxygenation. Despite apparent improvement in management and outcome of ARDS, the mortality rate in persons with the disease remains high, ranging from $35 \%$ to $65 \%$ (4).

Although mechanical ventilation provides essential life support, it can worsen lung injury (5). Computed tomography images of patients with ARDS show nonhomogeneous distribution of pulmonary aeration. Normally aerated lung regions are relatively small but, when they receive the largest part of tidal volume (VT) $(6,7)$, may be exposed to excessive alveolar wall tension and stress because of over-

See also:

Print

Editors' Notes. . 567

Web-Only

Appendix Tables

CME quiz

Conversion of graphics into slides distention $(8,9)$. Atelectatic lung regions are prone to cyclic recruitment and derecruitment, leading to shear stress in adjacent aerated and nonaerated alveoli (1012). Ventilator-induced lung injury is caused by excessive stress or strain to lung tissues that occurs during mechanical ventilation and aggravates inflammation and diffuse alveolar damage $(5,13)$.

Lung-protective ventilation strategies include ventilation with low VT and limited airway pressure to reduce ventilator-induced lung injury from overdistention while allowing hypercapnia and medium to high positive endexpiratory pressure (PEEP) to keep alveoli open throughout the ventilator cycle (14). Hypercapnia and acidosis may increase intracranial pressure, induce pulmonary hypertension, depress myocardial contractility, decrease renal blood flow, and release endogenous catecholamines (15). In addition, prevention of cyclic derecruitment with higher PEEP may contribute to overdistention of normally aerated alveoli, counterbalancing the benefits from low VT and limited airway pressure ventilation cycles (14).

The effect of different lung-protective ventilatory strategies in patients with acute lung injury or ARDS has been investigated in randomized, controlled trials (RCTs) testing higher versus lower $\mathrm{VT}_{\mathrm{T}}$ ventilation at similar PEEP (16-19), higher versus lower PEEP strategies during low VT ventilation (20-22), and lower VT and PEEP titrated 
greater than the lower inflection point of the individual pressure volume curve versus higher $\mathrm{VT}_{\mathrm{T}}$ and lower PEEP $(23,24)$. Results were partially conflicting because of differences in study design and number of enrolled patients. This may explain why most critically ill patients are still ventilated with high VT at lower or even no $\operatorname{PEEP}(4,25)$.

Our objective was to determine whether the different lung-protective ventilatory strategies improve outcome in critically ill adults with acute lung injury or ARDS.

\section{Methods}

\section{Data Sources and Searches}

We aimed to identify all RCTs assessing the efficacy and outcomes of lower VT ventilation, higher PEEP application, or a combination of both in adults with acute lung injury or ARDS. The electronic search strategy applied standard filters for identification of RCTs. We searched the Cochrane Central Register of Controlled Trials, MEDLINE (from inception to March 2009), and EMBASE (from inception to March 2009). Our search included the following keywords: acute lung injury, ALI, adult respiratory distress syndrome, ARDS, protective ventilation, lung protective ventilation strategy, pressure-limited ventilation, tidal volume, positive end-expiratory pressure, PEEP, and random. We did not apply language restrictions. In addition to the electronic search, we checked out cross-references from original articles and reviews.

\section{Selection of Studies}

We restricted the analysis to RCTs to guarantee control of selection bias. We did not include study designs containing inadequately adjusted planned co-interventions and quasirandomized or crossover trials. We considered RCTs that reported mortality as a predefined end point and compared lower versus higher VT ventilation, lower versus higher PEEP application, or a combination of these strategies in intubated and mechanically ventilated critically ill adults with acute lung injury or ARDS from any cause. Acute lung injury and ARDS had to be defined by the American-European Consensus Conference criteria (26) or by the Lung Injury Severity Score (27). Trials with a low VT ventilation strategy had to use lower VT, maximal inspiratory plateau pressure (PEI) of $30 \mathrm{~cm}$ $\mathrm{H}_{2} \mathrm{O}$ or less, or a combination, which resulted in VT of 8 $\mathrm{mL} / \mathrm{kg}$ of body weight or less, compared with conventional mechanical ventilation that used $\mathrm{VT}_{\mathrm{T}}$ ranging between 10 and $15 \mathrm{~mL} / \mathrm{kg}$. Regardless of the strategy used to deliver the lower $\mathrm{VT}$, the 2 study groups had to differ only for $\mathrm{VT}$ and not for other variables associated with a low VT ventilation strategy. Trials with high PEEP ventilation strategies had to use higher PEEP based on $\mathrm{FiO}_{2}-\mathrm{PEEP}$ scales, titrating PEEP to greater than the lower inflection point of the individual static or quasi-static pressure volume curve at enrollment or titrating PEEP as high as possible without increasing the maximal PEI to greater than $30 \mathrm{~cm} \mathrm{H}_{2} \mathrm{O}$ compared with conventional mechanical ventilation that used lower PEEP based on fixed $\mathrm{FiO}_{2}-\mathrm{PEEP}$ scales or lower PEEP at higher $\mathrm{FiO}_{2}$ to ensure

\section{Context}

Ventilation strategies to protect the lungs of patients with the acute respiratory distress syndrome (ARDS) include low tidal volume, limited airway pressures, and medium to high positive end-expiratory pressure (PEEP), but the adoption of these strategies has been slow in some clinical settings.

\section{Contribution}

This review of randomized trial evidence for low tidal volume and high PEEP ventilation on mortality of patients with ARDS or acute lung injury found that trials were limited in number but showed mortality benefits with lower versus higher tidal volume. High PEEP did not improve mortality in unselected patients but may help patients with life-threatening hypoxemia despite other interventions.

\section{Implication}

Lower tidal volume ventilation strategies should be used for patients with ARDS or acute lung injury.

—The Editors

adequate arterial oxygenation. We excluded studies in postoperative patients and those published only in abstract form. We contacted authors to clarify details of trials when necessary.

\section{Outcome Measures}

The primary outcome was mortality, evaluated at hospital discharge. Secondary outcomes included mortality at the end of the planned follow-up, barotrauma, use of rescue therapies owing to life-threatening hypoxemia, ventilator settings, and pulmonary function variables. Barotrauma was defined as any new pneumothorax, pneumomediastinum, subcutaneous emphysema, or pneumatocele after random assignment.

\section{Data Extraction and Quality Assessment}

Two pairs of independent reviewers performed the initial selection by screening titles and abstracts. Citations were selected for further evaluation if the studies they referred to were RCTs of lung-protective ventilatory strategies in critical ill adults or if the title or abstract did not give enough information to make an assessment. For detailed evaluation, we obtained the full text of all possibly relevant studies. Data from each study were extracted independently by the paired reviewers by using a prestandardized data abstraction form. One pair of reviewers was not informed about authors, journal, institutional affiliation, and date of publication. Data extracted from the publications were checked by another reviewer for accuracy. Quality assessment of these studies included use of randomization, reporting of allocation concealment, blinding, adequate selection and description of study population with respect to inclusion and exclusion criteria, similarity of the groups at baseline, use of a predefined treatment protocol, absence of confounders, absence of co-interventions, a priori definition of primary and secondary outcome variables, use of intention-to-treat analysis, extent of follow-up, a priori calcu- 
lation of sample size, number of patients screened and included in the trial, reports on patients lost to follow-up, and planned or premature termination of the RCT. Two reviewers independently used these criteria to abstract trial quality. We resolved any disagreements by consensus in consultation with a third reviewer if needed.

\section{Data Synthesis and Analysis}

We studied the following comparisons: lower versus higher VT ventilation using similar PEEP strategies, lower versus higher PEEP level during low VT ventilation, and the combination of higher $\mathrm{VT}_{\mathrm{T}}$ and lower PEEP level versus lower $\mathrm{VT}_{\mathrm{T}}$ and higher PEEP level.

\section{Qualitative Analysis}

We used a narrative summary approach to describe study characteristics and variation in quality indicators among studies and to consider how these factors affect our understanding of the outcomes of the RCTs included in the Cochrane review $(28,29)$.

\section{Quantitative Analysis}

The meta-analysis was performed according to the Cochrane Collaboration guidelines (30). All statistical analyses were performed with Review Manager, version 4.2 (The Nordic Cochrane Center, Copenhagen, Denmark), the Cochrane Collaboration's software for preparing and maintaining Cochrane systematic reviews (30). The pooled effects estimates for binary variables were expressed as odds ratios with 95\% CIs, whereas continuous variables were expressed as weighted mean differences with 95\% CIs. We tested the difference in estimates of treatment effect between the treatment and control groups for each hypothesis by using a 2 -sided $z$ test with statistical significance considered at a $P$ value of less than 0.05 . We examined heterogeneity by using the Cochran $Q$ and the $I^{2}$ test $(31,32)$. We predefined heterogeneity as low, moderate, and high, with $I^{2}$ statistics greater than $25 \%, 50 \%$, and $75 \%$, respectively (32). Meta-analysis with a random-effects model was applied with $I^{2}$ statistics greater than 25\% (33). Otherwise, we performed meta-analysis by using a fixed-effects model. However, the possibility of a type II (false-negative) error must be considered, and we made a thorough attempt to identify clinical heterogeneity or sources of bias. We considered a 1-tailed $P$ value less than 0.05 to be significant.

Interobserver agreement on selection of articles for inclusion and quality assessment was measured by using the Cohen (unweighted) $\kappa$ statistic (34). We considered a $\kappa$ value greater than 0.8 to indicate acceptable agreement.

\section{Role of the Funding Source}

We received no funding for this study.

\section{RESULTS}

\section{Study Selection}

Our initial electronic and manual search identified 1111 studies. Of these, we excluded 886 articles because they were not RCTs, did not evaluate lung-protective ventilatory strategies in patients with acute lung injury or ARDS, were duplicated references, or were not relevant. We retrieved 225 studies for more detailed analysis and excluded 216 of these (Figure). The 2 reviewer teams completely agreed $(\kappa=1)$ on the selection of included studies.

\section{Study Description}

Table 1 summarizes the study selection process, and Appendix Table 1 (available at www.annals.org) summarizes the characteristics of the included RCTs. All studies were multicenter trials (16-24). We identified definition of severity of patient lung injury, VT and PEI, $\mathrm{pH}$ thresholds and management of acidosis, use of recruitment maneuvers, use of rescue therapies, weaning procedure, and termination of the trials as key sources of between-study variation. Qualitative analysis of key study characteristics and quality indicators revealed the following differences.

\section{Definition of Severity of Patient Lung Injury}

Acute lung injury and ARDS were defined according to the American-European Consensus Conference criteria (26) in 6 RCTs $(16,18,20-22,24)$ and according to the Lung Injury Severity Score in 2 RCTs (17, 23). Although 1 study used the definition risk for ARDS (19), all patients fulfilled the Lung Injury Severity Score criteria for ARDS. Patients with ARDS or acute lung injury were investigated in 6 RCTs $(16-18,20-22)$, and patients with ARDS only were investigated in 3 studies (19, 23, 24). Seven RCTs reported Acute Physiology and Chronic Health Evaluation score (16, 18 $21,23,24)$, and 2 RCTs reported Simplified Acute Physiology Score II $(17,22)$.

\section{$V_{T}$ and $P_{E I}$}

Four RCTs tested lower versus higher VT ventilation at similar PEEP in 1149 patients in total (16-19), 3 RCTs compared lower versus higher PEEP values at low VT ventilation in 2299 patients in total (20-22), and 2 RCTs compared a combination of higher $\mathrm{VT}_{\mathrm{T}}$ and lower PEEP value versus lower $\mathrm{VT}$ and higher PEEP value in 148 patients in total $(23,24)$. Tidal volume was adjusted to actual body weight in 2 studies $(17,23)$, ideal body weight in 2 RCTs $(18,19)$, and predicted body weight in 5 RCTs $(16,20-22,24)$ (Appendix Table 2, available at www.annals.org). Observed VT, PEEP, respiratory rates, and PEI are given in Appendix Table 2. All RCTs comparing lower versus higher $V_{T}$ ventilation per protocol did not restrict PEI of $30 \mathrm{~cm}$ $\mathrm{H}_{2} \mathrm{O}$ or less in the higher VT groups (16-19). Three RCTs demonstrating improved outcome with lower VT ventilation observed a PEI greater than $30 \mathrm{~cm} \mathrm{H}_{2} \mathrm{O}$ during higher $V_{T}$ ventilation $(16,23,24)$. Protocols for management of mechanical ventilation were used in all RCTs (16-24), thus minimizing performance bias. 


\section{pH Thresholds and Management of Acidosis}

The ARDS Network strategy was to increase respiratory rate, up to 35 breaths/min, in an attempt to increase alveolar ventilation and thus keep $\mathrm{pH}$ greater than 7.30. Variations in $\mathrm{VT}_{\mathrm{T}}$ up to $8 \mathrm{~mL} / \mathrm{kg}$ of predicted body weight and PEI greater than $30 \mathrm{~cm} \mathrm{H}_{2} \mathrm{O}$ were allowed, and administration of sodium bicarbonate was considered when $\mathrm{pH}$ decreased to less than $7.15(16,20,22)$. If $\mathrm{pH}$ decreased to less than a defined threshold, an increased PEI greater than $30 \mathrm{~cm} \mathrm{H}_{2} \mathrm{O}$ was allowed in 4 RCTs $(16,19$, 20, 22), and administration of sodium bicarbonate was required in 7 RCTs (16-22) (Appendix Table 3, available at www.annals.org). $\mathrm{pH}$ thresholds ranged from 7.00 to 7.30 (16-23), and were not specified in 1 RCT (24), thus leaving $\mathrm{pH}$ management up to the clinician. Three RCTs defined a $\mathrm{pH}$ of 7.3 as the threshold requiring intervention $(18,20,22)$, whereas 5 studies set the threshold at 7.2 (23) or less than $7.2(16,17,19,21)$. The lowest $\mathrm{pH}$ thresholds of 7.00 (19) and 7.05 (17) were in 2 nonbeneficial RCTs, whereas the remaining 2 RCTs defined a threshold of 7.1 (21) and 7.15 (16) (Appendix Table 3).

\section{Use of Recruitment Maneuvers}

Recruitment maneuvers were regularly used in 2 RCTs (20, 23). In 1 RCT, only the first 80 patients randomly assigned to higher PEEP values received recruitment maneuvers (20).

\section{Use of Rescue Therapies}

Rescue therapies for refractory hypoxemia were predefined in 2 RCTs $(21,22)$. Rescue therapies were prone ventilation in 2 studies $(21,22)$, inhaled nitric oxide in 3 studies $(17,21,22)$, high-frequency oscillation in 1 study (21), intravenous almitrine bismesylate in 1 study (22), and extracorporeal membrane oxygenation in 1 study (21).

\section{Weaning Procedure}

Five studies used weaning protocols (16, 20-23).

\section{Termination of the Trial}

Only 2 RCTs were terminated after the planned estimated sample was enrolled $(19,21)$. Three RCTs were stopped prematurely after an interim analysis demonstrated futility $(17,20,22)$. One RCT was terminated early because the center participated in another trial (18). One RCT comparing lower versus higher $\mathrm{VT}_{\mathrm{T}}$ ventilation at similar PEEP (16) and 2 RCTs comparing a higher VT and lower PEEP ventilation strategy versus a lower $V_{T}$ and higher PEEP ventilation strategy $(23,24)$ were stopped prematurely after interim analyses demonstrated lower mortality in the lower $\mathrm{V}_{\mathrm{T}}$ groups. The mean duration of the individual RCTs was 33.3 months, ranging from 15 months (19) to 68 months (21). Only 3 RCTs reported on patients lost to follow-up $(21,22,24)$ (Table 1).
Figure. Literature search and selection.

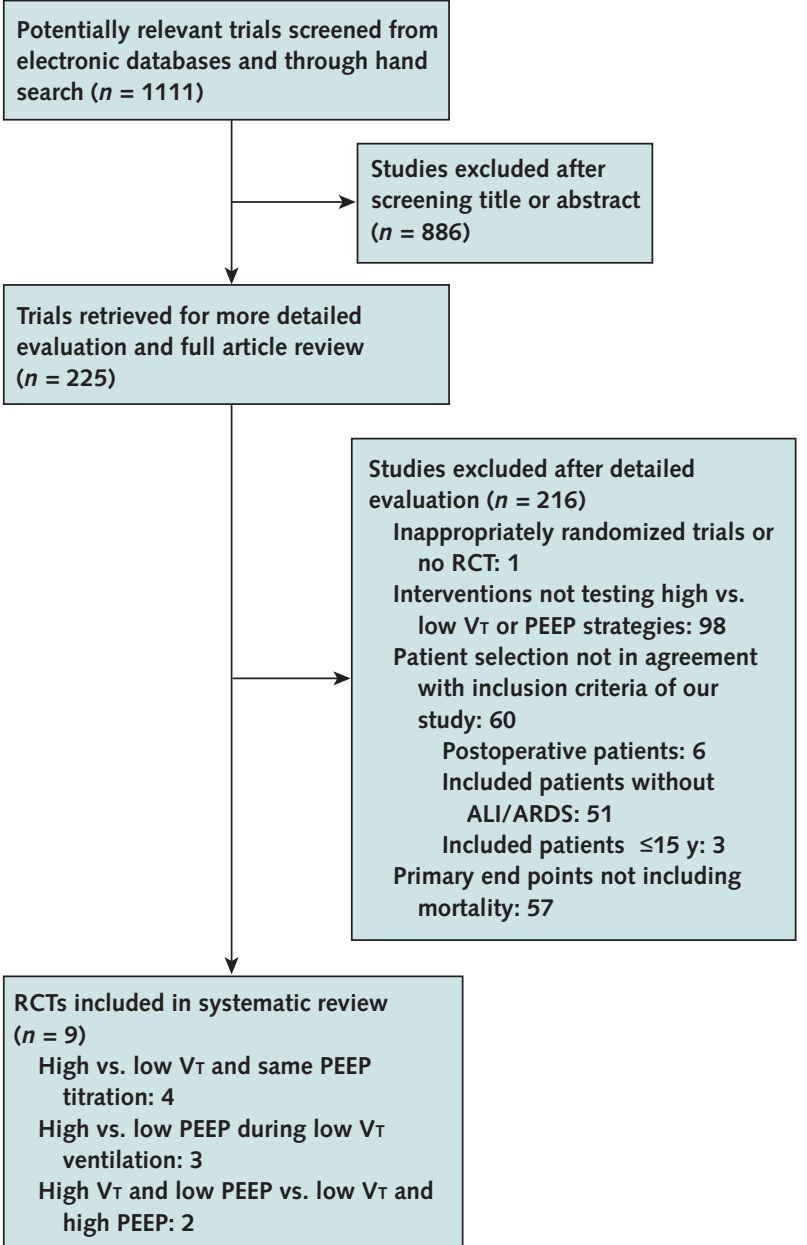

$\mathrm{ALI}=$ acute lung injury; ARDS = acute respiratory distress syndrome; PEEP $=$ positive end-expiratory pressure; $\mathrm{RCT}=$ randomized, controlled trial; $\mathrm{VT}_{\mathrm{T}}=$ tidal volume.

These differences among studies partially affect the overall strength of the evidence. To minimize the effects of study variation and optimize comparisons among ventilation strategies, we grouped RCTs on the basis of lower versus higher VT ventilation by using similar PEEP strategies, lower versus higher PEEP strategies during low $\mathrm{VT}_{\mathrm{T}}$ ventilation, and the combination of higher $\mathrm{VT}$ and lower PEEP strategies versus lower VT and higher PEEP strategies. In comparing lower versus higher $\mathrm{VT}_{\mathrm{T}}$ ventilation plus similar PEEP strategies and the combination of higher $\mathrm{VT}_{\mathrm{T}}$ and lower PEEP strategies versus lower $\mathrm{VT}_{\mathrm{T}}$ and higher PEEP strategies, the possible main confounding effect was the main goal of ventilator treatment on respiratory variables and clinical management of $\mathrm{pH}$.

\section{Evidence Synthesis}

Lower Versus Higher $V_{T}$ Ventilation Using Similar PEEP Values

The study characteristics that may explain differences in the benefit of lower VT ventilation were premature ter- 
Table 1. Characteristics of the Included Studies

\begin{tabular}{|c|c|c|c|c|c|c|}
\hline $\begin{array}{l}\text { Author, Year } \\
\text { (Reference) }\end{array}$ & $\begin{array}{l}\text { Random } \\
\text { Assignment }\end{array}$ & Allocation Concealment & Blinding & $\begin{array}{l}\text { Adequate } \\
\text { Selection and } \\
\text { Description of } \\
\text { Study Population }\end{array}$ & $\begin{array}{l}\text { Comparability of Groups (Baseline } \\
\text { Characteristics) }\end{array}$ & $\begin{array}{l}\text { Predefined } \\
\text { Treatment } \\
\text { Protocol }\end{array}$ \\
\hline \multicolumn{7}{|c|}{$\begin{array}{l}\text { Lower vs. higher } \mathrm{V}_{\mathrm{T}} \text { at } \\
\text { similar PEEP }\end{array}$} \\
\hline $\begin{array}{l}\text { Brochard et al, } \\
1998 \text { (17) }\end{array}$ & Yes & Yes, by sealed envelopes & No & Yes & $\begin{array}{l}\text { Yes, statistically proven for age, sex, APACHE } \\
\text { score, SAPS II score, previous duration of } \\
\mathrm{MV} \text {, LISS, } \mathrm{PaO}_{2}-\mathrm{FiO}_{2} \text { ratio, multiple trauma, } \\
\text { and immunosuppression. }\end{array}$ & Yes \\
\hline $\begin{array}{c}\text { Brower et al, } \\
1999 \text { (18) }\end{array}$ & Yes & Yes, not specified & No & Yes & $\begin{array}{l}\text { Yes, for age, sex, ethnic group, APACHE III } \\
\text { score, LISS, conditions causing ARDS, and } \\
\text { comorbid conditions. No } P \text { values given. }\end{array}$ & Yes \\
\hline $\begin{array}{l}\text { Brower et al, } \\
2000(16)\end{array}$ & Yes & $\begin{array}{l}\text { Yes, by } \\
\text { computer-generated } \\
\text { random list }\end{array}$ & No & Yes & $\begin{array}{l}\text { Not for minute ventilation. Statistically proven } \\
\text { for age, sex, ethnic group, APACHE III } \\
\text { score, } \mathrm{PaO}_{2}-\mathrm{FiO}_{2} \text { ratio, } \mathrm{VT}, \dot{\mathrm{V}}_{\mathrm{E}} \text {, number of } \\
\text { organ failures, and cause of lung injury. }\end{array}$ & Yes \\
\hline $\begin{array}{c}\text { Stewart et al, } \\
1998(19)\end{array}$ & Yes & $\begin{array}{l}\text { Yes, by } \\
\text { computer-generated } \\
\text { random list }\end{array}$ & No & Yes & $\begin{array}{l}\text { Not for } \mathrm{PaO}_{2}-\mathrm{FiO}_{2} \text { ratio. Statistically proven } \\
\text { for age, sex, APACHE II score, oxygen } \\
\text { index, MODS score, and number of risk } \\
\text { factors for ARDS. }\end{array}$ & Yes \\
\hline
\end{tabular}

\begin{tabular}{|c|c|c|c|c|c|c|}
\hline \multicolumn{7}{|c|}{$\begin{array}{l}\text { Lower vs. higher PEEP } \\
\text { at low } \mathrm{V}_{T}\end{array}$} \\
\hline $\begin{array}{l}\text { Brower et al, } \\
2004(20)\end{array}$ & Yes & $\begin{array}{l}\text { Yes, by } \\
\text { computer-generated } \\
\text { random list }\end{array}$ & No & Yes & $\begin{array}{l}\text { Not for age or } \mathrm{PaO}_{2}-\mathrm{FiO}_{2} \text { ratio. Statistically } \\
\text { proven for sex, ethnic group, APACHE III } \\
\text { score, } \mathrm{V}_{T}, \dot{\mathrm{V}}_{\mathrm{E}} \text {, respiratory rate, number of } \\
\text { organ failures, and cause of lung injury. }\end{array}$ & Yes \\
\hline $\begin{array}{l}\text { Meade et al, } \\
2008(21)\end{array}$ & Yes & $\begin{array}{l}\text { Yes, by } \\
\text { computer-generated } \\
\text { random list }\end{array}$ & No & Yes & $\begin{array}{l}\text { Not for age and incidence of sepsis. } \\
\text { Statistically proven for sex, duration of } \\
\text { hospital stay and } \mathrm{MV} \text {, APACHE II score, } \\
\text { MODS score, } \mathrm{PaO}_{2}-\mathrm{FiO}_{2} \text { ratio, oxygenation } \\
\text { index, PEEP, } \mathrm{PEI}, \mathrm{VT}_{\mathrm{T}}, \dot{\mathrm{V}}_{\mathrm{E}} \text {, respiratory rate, } \\
\text { barotrauma, and cause of lung injury. }\end{array}$ & Yes \\
\hline $\begin{array}{c}\text { Mercat et al, } \\
2008(22)\end{array}$ & Yes & $\begin{array}{l}\text { Yes, by } \\
\text { computer-generated } \\
\text { random list }\end{array}$ & No & Yes & $\begin{array}{l}\text { Yes, statistically proven for age, sex, SAPS II } \\
\text { score, septic shock, number of organ } \\
\text { failures, time since onset of ARDS, } \mathrm{V}_{\mathrm{T}}, \dot{\mathrm{V}}_{\mathrm{E}} \text {, } \\
\text { respiratory rate, PEEP, PEI, compliance, } \\
\mathrm{PaO}_{2}-\mathrm{FiO}_{2} \text { ratio, and cause of lung injury. }\end{array}$ & Yes \\
\hline
\end{tabular}

Lower $V_{T}+$ higher

PEEP vs. higher

$\mathrm{V}_{\mathrm{T}}+$ lower PEEP

Amato et al,

1998 (23)
Yes Yes, by sealed envelopes No Yes

Yes, by sealed envelopes No Yes
Villar et al,

2006 (24)
Yes

Yes
Yes, by sealed envelopes No
Yes proven for sex, ethnic group, APACHE organ $V_{T,} V_{E}$, respiratory rate, number of

Statistically proven for sex, duration of hospital stay and MV, APACHE II score, ODS score, $\mathrm{PaO}_{2}-\mathrm{FiO}_{2}$ ratio, oxygenation barotrauma, and cause of lung injury.

ctatistically proven for age, sex, SAPS II $\mathrm{PaO}_{2}-\mathrm{FiO}_{2}$ ratio, and cause of lung injury.
Predefined reatment 


\section{Table 1-Continued}

\begin{tabular}{|c|c|c|c|c|c|c|c|c|}
\hline $\begin{array}{l}\text { Absence of } \\
\text { Confounders }\end{array}$ & $\begin{array}{l}\text { Absence of } \\
\text { Co-interventions }\end{array}$ & $\begin{array}{l}\text { Outcome } \\
\text { Defined } \\
\text { A Priori }\end{array}$ & $\begin{array}{l}\text { Intention-to- } \\
\text { Treat } \\
\text { Analysis } \\
\text { Done }\end{array}$ & $\begin{array}{l}\text { Power } \\
\text { Analysis } \\
\text { Done }\end{array}$ & $\begin{array}{l}\text { Follow-up } \\
\text { Duration }\end{array}$ & $\begin{array}{l}\text { Patients } \\
\text { Screened/Included } \\
\text { in Trial, } n / n\end{array}$ & $\begin{array}{l}\text { Reported on } \\
\text { Patients } \\
\text { Lost to } \\
\text { Follow-up }\end{array}$ & $\begin{array}{l}\text { End of Study } \\
\text { (Duration of Study) }\end{array}$ \\
\hline
\end{tabular}

\begin{tabular}{|c|c|c|c|c|c|c|c|c|}
\hline Yes & No (iNO) & Yes & Yes & Yes & $60 \mathrm{~d}$ & $N R / 116$ & No & $\begin{array}{l}\text { Terminated early } \\
\text { because of futility } \\
\text { (32 mo) }\end{array}$ \\
\hline $\begin{array}{c}\text { No (AIDS, bone } \\
\text { marrow } \\
\text { transplant, } \\
\text { cancer) }\end{array}$ & Yes & Yes & Yes & Yes & $\begin{array}{l}28 \text { d, successful } \\
\text { weaning, or } \\
\text { death }\end{array}$ & $N R / 52$ & No & $\begin{array}{l}\text { Terminated early for } \\
\text { participation in } \\
\text { another trial ( } 22 \\
\text { mo) }\end{array}$ \\
\hline Yes & No (ketoconazole) & Yes & Yes & Yes & $\begin{array}{l}180 \mathrm{~d} \text { or hospital } \\
\text { discharge }\end{array}$ & NR/861 & No & $\begin{array}{l}\text { Terminated early } \\
\text { because of } \\
\text { efficacy ( } 24 \mathrm{mo})\end{array}$ \\
\hline Yes & Yes & Yes & Yes & NR & $\begin{array}{l}\text { Until hospital } \\
\text { discharge }\end{array}$ & $N R / 120$ & No & As planned (15 mo) \\
\hline
\end{tabular}

\begin{tabular}{|c|c|c|c|c|c|c|c|c|}
\hline Yes & No (RM) & Yes & Yes & Yes & $\begin{array}{l}90 \text { d or hospital } \\
\text { discharge }\end{array}$ & NR/549 & No & $\begin{array}{l}\text { Terminated early } \\
\text { because of futility } \\
(17 \mathrm{mo})\end{array}$ \\
\hline Yes & $\begin{array}{l}\text { No (iNO, HFOV, } \\
\text { ECMO, prone } \\
\text { positioning, } \\
\text { RM) }\end{array}$ & Yes & Yes & Yes & $\begin{array}{l}\text { Until hospital } \\
\text { discharge }\end{array}$ & NR/983 & $\begin{array}{l}\text { Yes, none } \\
\text { lost }\end{array}$ & As planned (68 mo) \\
\hline
\end{tabular}

\begin{tabular}{|c|c|c|c|c|c|c|c|c|}
\hline Yes & $\begin{array}{l}\text { No (iNO, prone } \\
\text { positioning, } \\
\text { RM, almitrine } \\
\text { bismesylate) }\end{array}$ & Yes & Yes & Yes & $60 \mathrm{~d}$ or death & $3429 / 767$ & $\begin{array}{l}\text { Yes, } 1 \\
\text { patient } \\
\text { lost }\end{array}$ & $\begin{array}{l}\text { Terminated early } \\
\text { because of futility } \\
\text { (40 mo) }\end{array}$ \\
\hline
\end{tabular}

\begin{tabular}{|c|c|c|c|c|c|c|c|c|}
\hline $\begin{array}{l}\text { No (leptospirosis, } \\
\text { iatrogenic } \\
\text { death) }\end{array}$ & No (RM) & Yes & Yes & Yes & $N R, 28 d$ & NR/53 & No & $\begin{array}{l}\text { Terminated early } \\
\text { because of } \\
\text { efficacy }(56 \mathrm{mo})\end{array}$ \\
\hline
\end{tabular}

\begin{tabular}{|c|c|c|c|c|c|c|c|}
\hline Yes & Yes & Yes $\quad$ Yes & Yes & $\mathrm{NR}, 30 \mathrm{~d}$ & $311 / 95$ & $\begin{array}{l}\text { Yes, none } \\
\text { lost }\end{array}$ & $\begin{array}{l}\text { Terminated early } \\
\text { because of } \\
\text { efficacy ( } 24 \mathrm{mo})\end{array}$ \\
\hline
\end{tabular}

19). None of the RCTs reported the number of patients requiring treatment of respiratory acidosis. A protocol for weaning was used only in the beneficial RCT (16).

Lower VT ventilation reduced the risk for hospital mortality (odds ratio, 0.75 [95\% CI, 0.58 to $0.96\{P=$ $\left.0.02\}] ; I^{2}=18.3 \%[P=0.29]\right)$, but not death at the end of follow-up (odds ratio, 0.94 [CI, 0.62 to 1.41 $\left.\{P=0.75\}] ; I^{2}=40.9 \%[P=0.17]\right)$ and barotrauma (odds ratio, 0.99 [CI, 0.68 to $1.46\{P=0.98\}] ; I^{2}=$ $0 \%[P=0.78]$ ) (Table 2). One study (16) that allowed higher VT ventilation, resulting in PEI greater than 30 $\mathrm{cm} \mathrm{H}_{2} \mathrm{O}$, carries $86 \%$ of the weight in the pooled effect and completely accounts for the heterogeneity and positive effect of lower VT ventilation on hospital mortality. When we excluded this study from analysis, no advantage of higher $\mathrm{VT}_{\mathrm{T}}$ ventilation could be demonstrated (odds ratio, 1.15 [CI, 0.63 to $2.09\{P=0.65\}] ; I^{2}=$ $0 \%[P=0.98])$. In the first 7 days, lower VT ventilation resulted in a lower $\mathrm{pH}$ at a lower PEI (Appendix Table 4, available at www.annals.org). Because of poorer oxygenation with lower VT ventilation, the PEEP value was higher than with high VT ventilation at day 1 (weighted mean difference, 0.71 [CI, 0.07 to $1.35\{P=0.03\}$ ]; $\left.I^{2}=45.8 \%[P=0.16]\right)$ (Appendix Table 4). 
REVIEW $\mid$ Ventilation Strategies and ARDS

\section{Table 2. Effect of Different Lung-Protective Ventilation Strategies on Mortality and Other End Points}

\begin{tabular}{|c|c|c|c|c|c|c|c|}
\hline \multirow[t]{2}{*}{$\begin{array}{l}\text { Author, Year (Reference), by Study } \\
\text { End Point }\end{array}$} & \multirow[t]{2}{*}{ Patients, $n$} & \multicolumn{2}{|c|}{$\begin{array}{l}\text { Patients With Study End Point, } \\
\text { by Ventilation Strategy, } n / n\end{array}$} & \multirow[t]{2}{*}{ Odds Ratio $(95 \% \mathrm{CI})$} & \multirow[t]{2}{*}{$P$ Value } & \multirow[t]{2}{*}{$\begin{array}{l}P^{2} \\
\text { Statistic }\end{array}$} & \multirow[t]{2}{*}{$P$ Value } \\
\hline & & $\begin{array}{l}\text { Low } V_{T} \text { at } \\
\text { Similar PEEP }\end{array}$ & $\begin{array}{l}\text { High } V_{T} \text { at } \\
\text { Similar PEEP }\end{array}$ & & & & \\
\hline \multicolumn{8}{|l|}{ Hospital mortality } \\
\hline Brochard et al, 1998 (17) & 116 & - & - & - & & & \\
\hline Brower et al, 1999 (18) & 52 & $13 / 26$ & $12 / 26$ & $1.17(0.39-3.47)^{*}$ & & & \\
\hline Brower et al, 2000 (16) & 861 & $134 / 432$ & $171 / 429$ & $0.68(0.51-0.90)^{*}$ & & & \\
\hline Stewart et al, 1998 (19) & 120 & $30 / 60$ & $28 / 60$ & $1.14(0.56-2.34)^{*}$ & & & \\
\hline \multirow[t]{2}{*}{ Summary } & & & & $0.75(0.58-0.96)^{*}$ & 0.020 & 18.3 & 0.29 \\
\hline & & $\begin{array}{l}\text { Low PEEP at } \\
\text { Low } V_{T}\end{array}$ & $\begin{array}{l}\text { High PEEP at } \\
\text { Low } V_{T}\end{array}$ & & & & \\
\hline Brower et al, 2004 (20) & 549 & $69 / 276$ & $75 / 273$ & $0.88(0.60-1.29)^{*}$ & & & \\
\hline Meade et al, 2008 (21) & 983 & $173 / 475$ & $205 / 508$ & $0.85(0.65-1.10)^{*}$ & & & \\
\hline Mercat et al, 2008 (22) & 767 & $136 / 385$ & $149 / 382$ & $0.85(0.64-1.15)^{*}$ & & & \\
\hline \multirow[t]{2}{*}{ Summary } & & & & $0.86(0.72-1.02)^{*}$ & 0.080 & 0 & 0.99 \\
\hline & & $\begin{array}{l}\text { Low } V_{T}+ \\
\text { High PEEP }\end{array}$ & $\begin{array}{l}\text { High } \mathrm{V}_{\mathrm{T}}+ \\
\text { Low PEEP }\end{array}$ & & & & \\
\hline Amato et al, 1998 (23) & 53 & $13 / 29$ & $17 / 24$ & $0.33(0.11-1.05)^{*}$ & & & \\
\hline Villar et al, 2006 (24) & 95 & $17 / 50$ & $25 / 45$ & $0.41(0.18-0.94)^{*}$ & & & \\
\hline \multirow[t]{2}{*}{ Summary } & & & & $0.38(0.20-0.75)^{*}$ & 0.005 & 0 & 0.77 \\
\hline & & $\begin{array}{l}\text { Low } \mathrm{V}_{\mathrm{T}} \text { at } \\
\text { Similar PEEP }\end{array}$ & $\begin{array}{l}\text { High } \mathrm{V}_{\mathrm{T}} \text { at } \\
\text { Similar PEEP }\end{array}$ & & & & \\
\hline \multicolumn{8}{|l|}{ Mortality at the end of follow-upt } \\
\hline Brochard et al, 1998 (17) & 116 & $27 / 58$ & $22 / 58$ & $1.43(0.68-2.99) \ddagger$ & & & \\
\hline Brower et al, 1999 (18) & 52 & $13 / 26$ & $12 / 26$ & $1.17(0.39-3.47) \ddagger$ & & & \\
\hline Brower et al, 2000 (16) & 861 & $134 / 432$ & $171 / 429$ & $0.68(0.51-0.90) \neq$ & & & \\
\hline Stewart et al, 1998 (19) & 120 & $30 / 60$ & $28 / 60$ & $1.14(0.56-2.34) \ddagger$ & & & \\
\hline Summary & & & & $0.94(0.62-1.41) \ddagger$ & 0.75 & 40.9 & 0.170 \\
\hline
\end{tabular}

\begin{tabular}{|c|c|c|c|c|c|c|c|}
\hline & & $\begin{array}{l}\text { Low PEEP at } \\
\text { Low } V_{T}\end{array}$ & $\begin{array}{l}\text { High PEEP at } \\
\text { Low } V_{T}\end{array}$ & & & & \\
\hline Brower et al, 2004 (20) & 549 & $69 / 276$ & $75 / 273$ & $0.88(0.60-1.29)^{*}$ & & & \\
\hline Meade et al, 2008 (21) & 983 & $173 / 475$ & $205 / 508$ & $0.85(0.65-1.10)^{*}$ & & & \\
\hline Mercat et al, 2008 (22) & 767 & $107 / 385$ & $119 / 382$ & $0.85(0.62-1.16)^{*}$ & & & \\
\hline \multirow[t]{2}{*}{ Summary } & & & & $0.85(0.72-1.02)^{*}$ & 0.080 & 0 & 0.99 \\
\hline & & $\begin{array}{l}\text { Low } V_{T}+ \\
\text { High PEEP }\end{array}$ & $\begin{array}{l}\text { High } V_{T}+ \\
\text { Low PEEP }\end{array}$ & & & & \\
\hline Amato et al, 1998 (23) & 53 & $11 / 29$ & $17 / 24$ & $0.25(0.08-0.80)^{*}$ & & & \\
\hline Villar et al, 2006 (24) & 95 & $16 / 50$ & $24 / 45$ & $0.41(0.18-0.95)^{*}$ & & & \\
\hline \multirow[t]{2}{*}{ Summary } & & & & $0.35(0.18-0.68)^{*}$ & 0.002 & 0 & 0.50 \\
\hline & & $\begin{array}{l}\text { Low } \mathrm{V}_{\mathrm{T}} \text { at } \\
\text { Similar PEEP }\end{array}$ & $\begin{array}{l}\text { High } V_{T} \text { at } \\
\text { Similar PEEP }\end{array}$ & & & & \\
\hline \multicolumn{8}{|l|}{ Barotrauma } \\
\hline Brochard et al, 1998 (17) & 116 & $8 / 58$ & $7 / 58$ & $1.17(0.39-3.46)^{*}$ & & & \\
\hline Brower et al, 1999 (18) & 52 & $2 / 26$ & $1 / 26$ & $2.08(0.18-24.51)^{*}$ & & & \\
\hline Brower et al, 2000 (16) & 861 & $43 / 432$ & $47 / 429$ & $0.90(0.58-1.39)^{*}$ & & & \\
\hline Stewart et al, 1998 (19) & 120 & $6 / 60$ & $4 / 60$ & $1.56(0.42-5.82)^{*}$ & & & \\
\hline \multirow[t]{2}{*}{ Summary } & & & & $0.99(0.68-1.46)^{*}$ & 0.98 & 0 & 0.78 \\
\hline & & $\begin{array}{l}\text { Low PEEP at } \\
\text { Low } V_{T}\end{array}$ & $\begin{array}{l}\text { High PEEP at } \\
\text { Low } V_{T}\end{array}$ & & & & \\
\hline Brower et al, 2004 (20) & 549 & $30 / 276$ & $27 / 273$ & $1.11(0.64-1.92)^{*}$ & & & \\
\hline Meade et al, 2008 (21) & 983 & $53 / 475$ & $47 / 508$ & $1.23(0.81-1.86)^{*}$ & & & \\
\hline Mercat et al, 2008 (22) & 767 & $26 / 385$ & $22 / 382$ & $1.19(0.66-2.13)^{*}$ & & & \\
\hline \multirow[t]{2}{*}{ Summary } & & & & $1.19(0.89-1.58)^{*}$ & 0.25 & 0 & 0.96 \\
\hline & & $\begin{array}{l}\text { Low } V_{T}+ \\
\text { High PEEP }\end{array}$ & $\begin{array}{l}\text { High } V_{T}+ \\
\text { Low PEEP }\end{array}$ & & & & \\
\hline Amato et al, 1998 (23) & 53 & $2 / 29$ & $10 / 24$ & $0.10(0.02-0.54)^{*}$ & & & \\
\hline Villar et al, 2006 (24) & 95 & $2 / 50$ & $4 / 45$ & $0.43(0.07-2.45)^{*}$ & & & \\
\hline Summary & & & & $0.20(0.06-0.63)^{*}$ & 0.006 & 25.1 & 0.25 \\
\hline
\end{tabular}




\begin{tabular}{|c|c|c|c|c|c|c|c|}
\hline \multirow[t]{2}{*}{$\begin{array}{l}\text { Author, Year (Reference), by Study End } \\
\text { Point }\end{array}$} & \multirow[t]{2}{*}{ Patients, $n$} & \multicolumn{2}{|c|}{$\begin{array}{l}\text { Patients With Study End Point, } \\
\text { by Ventilation Strategy, } n / n\end{array}$} & \multirow[t]{2}{*}{ Odds Ratio $(95 \% \mathrm{Cl})$} & \multirow[t]{2}{*}{$P$ Value } & \multirow[t]{2}{*}{$\begin{array}{l}P^{2} \\
\text { Statistic }\end{array}$} & \multirow[t]{2}{*}{$P$ Value } \\
\hline & & $\begin{array}{l}\text { Low PEEP at } \\
\text { Low } V_{T}\end{array}$ & $\begin{array}{l}\text { High PEEP at } \\
\text { Low } V_{T}\end{array}$ & & & & \\
\hline \multicolumn{8}{|l|}{ Use of rescue therapy } \\
\hline Meade et al, 2008 (21) & 983 & $37 / 475$ & $61 / 508$ & $0.62(0.40-0.95) \neq$ & & & \\
\hline Mercat et al, 2008 (22) & 767 & $72 / 385$ & $132 / 382$ & $0.44(0.31-0.61) \ddagger$ & & & \\
\hline Summary & & & & $0.51(0.36-0.71) \ddagger$ & $<0.001$ & 37.9 & 0.20 \\
\hline & & $\begin{array}{l}\text { Low PEEP at } \\
\text { Low } V_{T}\end{array}$ & $\begin{array}{l}\text { High PEEP at } \\
\text { Low } V_{T}\end{array}$ & & & & \\
\hline \multicolumn{8}{|l|}{ Mortality in patients with rescue therapy } \\
\hline Meade et al, 2008 (21) & 983 & $20 / 475$ & $45 / 508$ & $0.45(0.26-0.78)^{*}$ & & & \\
\hline Mercat et al, 2008 (22) & 767 & $37 / 385$ & $62 / 382$ & $0.55(0.36-0.85)^{*}$ & & & \\
\hline Summary & & & & $0.51(0.36-0.71)^{*}$ & $<0.001$ & 0 & 0.59 \\
\hline
\end{tabular}

PEEP $=$ positive end-expiratory pressure; $\mathrm{VT}_{\mathrm{T}}=$ tidal volume

* Fixed odds ratio.

† Primary study end point.

‡ Random odds ratio.

\section{Lower Versus Higher PEEP Value at Low VT Ventilation}

The relevant differences in study characteristics were premature termination, use of recruitment maneuvers, and rescue therapies. Two RCTs were stopped prematurely after interim analysis demonstrated futility $(20,22)$. Recruitment maneuvers were regularly used in the higher PEEP group in 1 RCT (21) and in another RCT (20), were used only in the first 80 patients assigned to higher PEEP. Two RCTs used rescue therapies more frequently for refractory hypoxemia in the lower PEEP group $(21,22)$.

Risk for hospital mortality (odds ratio, 0.86 [CI, 0.72 to $\left.1.02\{P=0.08\}] ; I^{2}=0 \%[P=0.99]\right)$ and barotrauma (odds ratio, 1.19 [CI, 0.89 to $1.58\{P=0.25\}] ; I^{2}=0 \%$ $[P=0.96])$ were similar between lower and higher PEEP values at low VT ventilation (Table 2). High PEEP value and lower $\mathrm{VT}$ ventilation reduced requirement of rescue therapies because of life-threatening hypoxemia (odds ratio, 0.51 [CI, 0.36 to $0.71\{P<0.001\}] ; I^{2}=37.9 \%[P=$ $0.20])$ and decreased mortality in patients who received rescue therapy (odds ratio, 0.51 [CI, 0.36 to $0.71\{P<$ $0.001\}] ; I^{2}=0 \%[P=0.59]$ ) (Table 2). In the first 7 days, higher PEEP resulted in better arterial oxygenation at higher PEI (Appendix Table 4).

\section{Combination of Higher $V_{T}$ and Lower PEEP Versus Lower $V_{T}$ and Higher PEEP}

Two RCTs $(23,24)$ had the greatest differences in VT and PEI between study groups $(23,24)$. One RCT (23) reported $70 \%$ mortality in the higher VT and lower PEEP group, which may be explained by a higher incidence of leptospirosis and iatrogenic death.

The combination of lower VT and higher PEEP reduced the risk for hospital mortality (odds ratio, 0.38 [CI, 0.20 to $\left.0.75\{P=0.005\}] ; I^{2}=0 \%[P=0.77]\right)$ and barotrauma (odds ratio, 0.20 [CI, 0.06 to $0.63\{P=0.006\}$ ]; $I^{2}=25.1 \%[P=0.25]$ ) (Table 2). In the first 7 days, lower VT and higher PEEP resulted in higher $\mathrm{PaCO}_{2}$ at a lower Pei (Appendix Table 4).

\section{Discussion}

Available evidence from a limited number of RCTs tends to show the following in patients with acute lung injury or ARDS: reduction in hospital mortality with lower VT ventilation, similar hospital mortality with higher or lower PEEP strategies using lower VT ventilation, and reduced need for rescue therapy and reduced mortality in patients receiving rescue therapies during higher PEEP ventilation with lower VT.

Previous systematic reviews and meta-analyses did not focus strictly on the comparison between lower and higher VT ventilation at similar PEEP; rather, they also included trials in which $\mathrm{VT}_{\mathrm{T}}$ was reduced at the same time that PEEP was markedly increased (35-37), and they did not evaluate recent RCTs comparing higher versus lower PEEP strategies with lower VT ventilation (Appendix Table 5, available at www.annals.org). Our meta-analysis was performed according to the Cochrane Collaboration guidelines (30) and included 9 RCTs with a total of 3596 patients and distinguished between lower versus higher VT ventilation using similar PEEP strategies, lower versus higher PEEP value at low $\mathrm{VT}_{\mathrm{T}}$ ventilation, and the combination of higher $\mathrm{VT}$ and lower PEEP versus lower VT and higher PEEP. Thus, our meta-analysis should better separate the effects of $\mathrm{VT}_{\mathrm{T}}$ and PEEP value on mortality.

Lower VT ventilation using similar PEEP strategies shows a relatively consistent significant reduction in mortality. Our finding supports the hypothesis that the higher heterogeneity found in previous meta-analysis can be partially attributed to the inclusion of RCTs that simultaneously investigated lower $\mathrm{VT}_{\mathrm{T}}$ and higher PEEP strategies (38). However, from a statistical standpoint, some uncer- 
tainty may still exist regarding the benefit of low $\mathrm{VT}$ on mortality. When the ARDS Network study (16), which carries $86 \%$ of the weight in the pooled effect and completely accounts for the heterogeneity, was excluded from analysis, no advantage of higher $\mathrm{VT}_{\mathrm{T}}$ ventilation could be demonstrated.

In agreement with previous reports (35-38), we found that lower VT ventilation did not improve outcome when higher $\mathrm{VT}$ ventilation resulted in PEI no greater than $30 \mathrm{~cm} \mathrm{H}_{2} \mathrm{O}$. However, none of our analyses demonstrated an advantage of high $\mathrm{VT}_{\mathrm{T}}$ ventilation. Thus, low VT ventilation seems to be beneficial in patients with acute lung injury or ARDS for routine clinical use if potential side effects, such as hypercapnia and respiratory acidosis, are not contraindicated.

Two RCTs that did not demonstrate an advantage of lower $\mathrm{VT}_{\mathrm{T}}$ ventilation accepted $\mathrm{pH}$ thresholds of 7.00 and 7.05 before increasing VT or administering sodium bicarbonate $(17,19)$. Although ventilation with lower VT was associated with lower $\mathrm{pH}$ and a trend toward higher $\mathrm{PaCO}_{2}$, mortality was not affected. Thus, lower $\mathrm{pH}$ or active treatment of respiratory acidosis should not have confounded the effects of lower VT ventilation.

Although all included RCTs (16-19) tested lower versus higher VT ventilation using similar PEEP strategies, PEEP was slightly but statistically significant higher (0.8 to $1.5 \mathrm{~cm} \mathrm{H}_{2} \mathrm{O}$ ) with lower VT ventilation on day 1. Poorer arterial oxygenation requiring higher $\mathrm{FiO}_{2}$ to maintain the targeted oxygenation goal resulted in higher PEEP in all applied PEEP strategies (16-19). Lower VT ventilation has been demonstrated to be associated with alveolar derecruitment and hence poorer oxygenation (39), which may explain the slightly higher $\mathrm{FiO}_{2}$ and PEEP values during lower VT ventilation on day 1 . Despite the slightly higher PEEP, lower VT ventilation resulted in a significantly lower PEI. Thus, the small differences in PEEP should not have confounded the beneficial effects of lower VT ventilation.

Ventilation with lower VT and PEI was not associated with reduced risk for barotrauma. Apparently, the higher VT used in the analyzed RCTs (16-19) did not result in great enough alveolar wall tension and stress to cause alveolar rupture and gross barotrauma. However, on the basis of the definitions of barotrauma that were used, minor structural damage cannot be excluded. It is generally believed that during higher VT ventilation, even moderate alveolar wall tension and stress may induce pulmonary and systemic inflammatory response, contributing to increased morbidity and mortality (12). Because only the ARDS Network study (16) reported blood concentrations of inflammatory mediators and incidence and severity of organ dysfunction, we could not systematically analyze the importance of the ventilation-induced pulmonary and systemic inflammatory response on outcome.

Despite the different criteria used for PEEP selection, 3 RCTs $(20-22)$ demonstrated no difference in mortality comparing lower versus higher PEEP value with lower VT ventilation. Thus, random application of either higher or lower PEEP strategy in an unselected population with acute lung injury or ARDS does not significantly improve outcome. The lack of benefit of higher PEEP strategies observed in RCTs (20-22) may be explained by inclusion of a substantial proportion of patients, in whom the extent of lung edema and collapse were modest (40). In 2 RCTs (21, 22 ), $7.8 \%$ to $34.6 \%$ of patients needed rescue therapies to prevent decrease in $\mathrm{PaO}_{2}$ less than $55 \mathrm{~mm} \mathrm{Hg}$ or in arterial oxygen saturation less than $88 \%$ at $\mathrm{FiO}_{2}$ of 0.80 or greater. Requirement of rescue therapies to prevent life-threatening hypoxemia and mortality in patients who received rescue therapy were reduced in the higher PEEP groups $(21,22)$. Thus, our results suggest that higher PEEP strategies may be beneficial to prevent life-threatening hypoxemia in patients with severe ARDS. Despite limitation of $\mathrm{VT}$, higher PEEP strategies improved arterial oxygenation and increased PEI, which may have contributed to overdistention of normally aerated alveoli, counterbalancing small possible benefits of higher PEEP in patients with less severe illness $(2,8,40,41)$. Increase in PEI during low VT ventilation was suggested be associated with a higher mortality risk (42). However, higher PEEP strategies did not result in great enough alveolar wall tension and stress to cause alveolar rupture and gross barotrauma when VT and PEI were limited. Of note, our data demonstrate that higher PEEP with PEI limited to no greater than $30 \mathrm{~cm} \mathrm{H}_{2} \mathrm{O}$ does not induce harm in an unselected population with acute lung injury or ARDS. To counteract possible cardiovascular depression caused by higher PEEP and PEI, frequent fluid loading associated with a positive fluid balance or vasopressors may be required, which has been shown to delay pulmonary recovery (43). Because the 3 RCTs $(21,22,43)$ did not consistently report fluid and vasopressor management, we could not systematically analyze the role of these factors on outcome.

On the basis of analysis of 2 RCTs including only 148 patients, the combination of lower $\mathrm{VT}_{\mathrm{T}}$ and higher PEEP reduced the risk for hospital mortality $(23,24)$. However, in Amato and coworkers' trial (23), mortality in the control group was $30 \%$ higher than that in similar studies. Both RCTs included patients with severe ARDS in whom higher PEEP strategies are expected to be more effective to prevent cyclic recruitment and derecruitment and hence ventilator-induced lung injury (44). Compared with ventilation with higher $\mathrm{V}_{\mathrm{T}}$ and lower PEEP, the simultaneous reduction in $\mathrm{VT}_{\mathrm{T}}$ and increase in PEEP resulted in a decrease in PEI and risk for gross barotrauma. The increase in barotrauma during ventilation with higher $\mathrm{VT}$ and lower PEEP may be explained by higher PEI that ranged on average between 32.6 and $34.4 \mathrm{~cm} \mathrm{H}_{2} \mathrm{O}$ and by increased tidal pressure amplitude. Both mechanisms could have led to excessive regional mechanical strain and stresses promoting structural parenchymal damage and clinically evident barotrauma (9). 
We conducted an extensive literature search to retrieve all relevant eligible trials and to minimize the potential for publication bias. However, we could retrieve only 9 eligible RCTs that used different lung-protective ventilatory strategies, and these were not easily comparable. To minimize the degree of heterogeneity among studies, we grouped the RCTs according to the specific lung-protective ventilatory strategies used. This resulted in a relatively small number of RCTs analyzed in each group. All RCTs (16-24) reported mortality as the primary outcome, and this was evaluated at different time points after randomization. We defined hospital mortality as primary outcome; this was reported by $8(16,18,19-24)$ of the $9(16-24)$ included RCTs. Because length of mechanical ventilation, hospital stay, or intensive care stay and incidence and severity of organ dysfunction were frequently not reported or not normally distributed, we could not include them in the analysis. In addition, length of mechanical ventilation was considered difficult to interpret because $4(17-19,23)$ of the 9 RCTs did not use standardized weaning protocols.

In conclusion, on the basis of available evidence from a limited number of RCTs, routine use of low VT tends to be beneficial in all patients with acute lung injury or ARDS because this ventilation strategy improved hospital mortality. Higher PEEP strategies during lower VT ventilation did not improve hospital mortality and cannot be recommended in unselected patients with acute lung injury or ARDS. Higher PEEP strategies during lower VT ventilation may prevent life-threatening hypoxemia.

From University Hospital Bonn, Bonn, Germany, and the University of Insubria, Varese, Italy.

Potential Conflicts of Interest: None disclosed.

Reproducible Research Statement: Study protocol and data set: Not available. Statistical code: Available from Dr. Zinserling (e-mail, joerg .zinserling@ukb.uni-bonn.de).

Requests for Single Reprints: Christian Putensen, MD, PhD, Department of Anaesthesiology and Intensive Care Medicine, University of Bonn, Sigmund-Freud-Strasse 25, 53105 Bonn, Germany; e-mail, putensen@buni-bonn.de.

Current author addresses and author contributions are available at www .annals.org.

\section{References}

1. Lennon M. Ashbaugh DG, Bigelow DB, Petty TL, Levine BE. Acute respiratory distress in adults. The Lancet, Saturday 12 August 1967. Crit Care Resusc. 2005;7:60-1. [PMID: 16548822]

2. Gattinoni L, Caironi P, Cressoni M, Chiumello D, Ranieri VM, Quintel M, et al. Lung recruitment in patients with the acute respiratory distress syndrome. N Engl J Med. 2006;354:1775-86. [PMID: 16641394]

3. Villar J, Pérez-Méndez L, López J, Belda J, Blanco J, Saralegui I, et al; HELP Network. An early PEEP/FiO ${ }_{2}$ trial identifies different degrees of lung injury in patients with acute respiratory distress syndrome. Am J Respir Crit Care Med. 2007;176:795-804. [PMID: 17585106]

4. Esteban A, Ferguson ND, Meade MO, Frutos-Vivar F, Apezteguia C, Bro- chard L, et al; VENTILA Group. Evolution of mechanical ventilation in response to clinical research. Am J Respir Crit Care Med. 2008;177:170-7. [PMID: 17962636]

5. Tremblay LN, Slutsky AS. Ventilator-induced lung injury: from the bench to the bedside. Intensive Care Med. 2006;32:24-33. [PMID: 16231069]

6. Gattinoni L, Pelosi P, Crotti S, Valenza F. Effects of positive end-expiratory pressure on regional distribution of tidal volume and recruitment in adult respiratory distress syndrome. Am J Respir Crit Care Med. 1995;151:1807-14. [PMID: 7767524]

7. Terragni PP, Rosboch G, Tealdi A, Corno E, Menaldo E, Davini O, et al. Tidal hyperinflation during low tidal volume ventilation in acute respiratory distress syndrome. Am J Respir Crit Care Med. 2007;175:160-6. [PMID: 17038660]

8. Rouby JJ, Puybasset L, Nieszkowska A, Lu Q. Acute respiratory distress syndrome: lessons from computed tomography of the whole lung. Crit Care Med. 2003;31:S285-95. [PMID: 12682454]

9. Chiumello D, Carlesso E, Cadringher P, Caironi P, Valenza F, Polli F, et al. Lung stress and strain during mechanical ventilation for acute respiratory distress syndrome. Am J Respir Crit Care Med. 2008;178:346-55. [PMID: 18451319] 10. Halter JM, Steinberg JM, Schiller HJ, DaSilva M, Gatto LA, Landas S, et al. Positive end-expiratory pressure after a recruitment maneuver prevents both alveolar collapse and recruitment/derecruitment. Am J Respir Crit Care Med. 2003;167:1620-6. [PMID: 12615628]

11. Marini JJ, Hotchkiss JR, Broccard AF. Bench-to-bedside review: microvascular and airspace linkage in ventilator-induced lung injury. Crit Care. 2003;7: 435-44. [PMID: 14624683]

12. Ranieri VM, Suter PM, Tortorella C, De Tullio R, Dayer JM, Brienza A, et al. Effect of mechanical ventilation on inflammatory mediators in patients with acute respiratory distress syndrome: a randomized controlled trial. JAMA. 1999; 282:54-61. [PMID: 10404912]

13. Tremblay LN, Slutsky AS. Pathogenesis of ventilator-induced lung injury: trials and tribulations [Editorial]. Am J Physiol Lung Cell Mol Physiol. 2005; 288:L596-8. [PMID: 15757952]

14. Pinhu L, Whitehead T, Evans T, Griffiths M. Ventilator-associated lung injury. Lancet. 2003;361:332-40. [PMID: 12559881]

15. Feihl F, Perret C. Permissive hypercapnia. How permissive should we be? Am J Respir Crit Care Med. 1994;150:1722-37. [PMID: 7952641]

16. Brower RG, Matthay MA, Morris A, Schoenfeld D, Thompson BT, Wheeler A; The Acute Respiratory Distress Syndrome Network. Ventilation with lower tidal volumes as compared with traditional tidal volumes for acute lung injury and the acute respiratory distress syndrome. N Engl J Med. 2000; 342:1301-8. [PMID: 10793162]

17. Brochard L, Roudot-Thoraval F, Roupie E, Delclaux C, Chastre J, Fernandez-Mondéjar E, et al. Tidal volume reduction for prevention of ventilator-induced lung injury in acute respiratory distress syndrome. The Multicenter Trail Group on Tidal Volume reduction in ARDS. Am J Respir Crit Care Med. 1998;158:1831-8. [PMID: 9847275]

18. Brower RG, Shanholtz CB, Fessler HE, Shade DM, White P Jr, Wiener $\mathrm{CM}$, et al. Prospective, randomized, controlled clinical trial comparing traditional versus reduced tidal volume ventilation in acute respiratory distress syndrome patients. Crit Care Med. 1999;27:1492-8. [PMID: 10470755]

19. Stewart TE, Meade MO, Cook DJ, Granton JT, Hodder RV, Lapinsky SE, et al. Evaluation of a ventilation strategy to prevent barotrauma in patients at high risk for acute respiratory distress syndrome. Pressure- and Volume-Limited Ventilation Strategy Group. N Engl J Med. 1998;338:355-61. [PMID: 9449728]

20. Brower RG, Lanken PN, MacIntyre N, Matthay MA, Morris A, Ancukiewicz $\mathrm{M}$, et al; National Heart, Lung, and Blood Institute ARDS Clinical Trials Network. Higher versus lower positive end-expiratory pressures in patients with the acute respiratory distress syndrome. N Engl J Med. 2004;351:327-36. [PMID: 15269312] 21. Meade MO, Cook DJ, Guyatt GH, Slutsky AS, Arabi YM, Cooper DJ, et al; Lung Open Ventilation Study Investigators. Ventilation strategy using low tidal volumes, recruitment maneuvers, and high positive end-expiratory pressure for acute lung injury and acute respiratory distress syndrome: a randomized controlled trial. JAMA. 2008;299:637-45. [PMID: 18270352]

22. Mercat A, Richard JC, Vielle B, Jaber S, Osman D, Diehl JL, et al; Expiratory Pressure (Express) Study Group. Positive end-expiratory pressure setting in adults with acute lung injury and acute respiratory distress syndrome: a randomized controlled trial. JAMA. 2008;299:646-55. [PMID: 18270353] 23. Amato MB, Barbas CS, Medeiros DM, Magaldi RB, Schettino GP, Lorenzi-Filho G, et al. Effect of a protective-ventilation strategy on mortality in the acute respiratory distress syndrome. N Engl J Med. 1998;338:347-54. 
[PMID: 9449727]

24. Villar J, Kacmarek RM, Pérez-Méndez L, Aguirre-Jaime A. A high positive end-expiratory pressure, low tidal volume ventilatory strategy improves outcome in persistent acute respiratory distress syndrome: a randomized, controlled trial. Crit Care Med. 2006;34:1311-8. [PMID: 16557151]

25. Sakr Y, Vincent JL, Reinhart K, Groeneveld J, Michalopoulos A, Sprung $\mathrm{CL}$, et al; Sepsis Occurence in Acutely Ill Patients Investigators. High tidal volume and positive fluid balance are associated with worse outcome in acute lung injury. Chest. 2005;128:3098-108. [PMID: 16304249]

26. Bernard GR, Artigas A, Brigham KL, Carlet J, Falke K, Hudson L, et al. The American-European Consensus Conference on ARDS. Definitions, mechanisms, relevant outcomes, and clinical trial coordination. Am J Respir Crit Care Med. 1994;149:818-24. [PMID: 7509706]

27. Murray JF, Matthay MA, Luce JM, Flick MR. An expanded definition of the adult respiratory distress syndrome. Am Rev Respir Dis. 1988;138:720-3. [PMID: 3202424]

28. Dixon-Woods M, Shaw RL, Agarwal S, Smith JA. The problem of appraising qualitative research. Qual Saf Health Care. 2004;13:223-5. [PMID: 15175495]

29. Denzin N, Riessman C. Narrative analysis. J Commun. 1995;45:177-84.

30. Higgins JP, Green S, eds. Cochrane Handbook for Systematic Reviews of Interventions. Version 4.2.6. The Cochrane Collaboration; 2006.

31. Higgins JP, Thompson SG. Quantifying heterogeneity in a meta-analysis. Stat Med. 2002;21:1539-58. [PMID: 12111919]

32. Higgins JP, Thompson SG, Deeks JJ, Altman DG. Measuring inconsistency in meta-analyses. BMJ. 2003;327:557-60. [PMID: 12958120]

33. Deeks JJ. Systematic reviews in health care: systematic reviews of evaluations of diagnostic and screening tests. BMJ. 2001;323:157-62. [PMID: 11463691]

34. Fleiss JL, Cohen J. The equivalence of weighted kappa and the intraclass correlation coefficient as measures of reliability. Educ Psychol Meas. 1973;33: 613-619.

35. Moran JL, Bersten AD, Solomon PJ. Meta-analysis of controlled trials of ventilator therapy in acute lung injury and acute respiratory distress syndrome: an alternative perspective. Intensive Care Med. 2005;31:227-35. [PMID: 15678318]
36. Petrucci N, Iacovelli W. Ventilation with smaller tidal volumes: a quantitative systematic review of randomized controlled trials. Anesth Analg. 2004;99: 193-200. [PMID: 15281529]

37. Petrucci N, Iacovelli W. Lung protective ventilation strategy for the acute respiratory distress syndrome. Cochrane Database Syst Rev. 2007:CD003844. [PMID: 17636739]

38. Eichacker PQ, Gerstenberger EP, Banks SM, Cui X, Natanson C. Metaanalysis of acute lung injury and acute respiratory distress syndrome trials testing low tidal volumes. Am J Respir Crit Care Med. 2002;166:1510-4. [PMID: 12406836]

39. Pelosi P, Goldner M, McKibben A, Adams A, Eccher G, Caironi P, et al. Recruitment and derecruitment during acute respiratory failure: an experimental study. Am J Respir Crit Care Med. 2001;164:122-30. [PMID: 11435250]

40. Gattinoni L, Caironi P. Refining ventilatory treatment for acute lung injury and acute respiratory distress syndrome [Editorial]. JAMA. 2008;299:691-3. [PMID: 18270359]

41. Vieira SR, Puybasset L, Richecoeur J, Lu Q, Cluzel P, Gusman PB, et al. A lung computed tomographic assessment of positive end-expiratory pressureinduced lung overdistension. Am J Respir Crit Care Med. 1998;158:1571-7. [PMID: 9817710]

42. Hager DN, Krishnan JA, Hayden DL, Brower RG; ARDS Clinical Trials Network. Tidal volume reduction in patients with acute lung injury when plateau pressures are not high. Am J Respir Crit Care Med. 2005;172:1241-5. [PMID: 16081547]

43. Wiedemann HP, Wheeler AP, Bernard GR, Thompson BT, Hayden D, deBoisblanc B, et al; National Heart, Lung, and Blood Institute Acute Respiratory Distress Syndrome (ARDS) Clinical Trials Network. Comparison of two fluid-management strategies in acute lung injury. N Engl J Med. 2006;354:256475. [PMID: 16714767]

44. Borges JB, Okamoto VN, Matos GF, Caramez MP, Arantes PR, Barros F, et al. Reversibility of lung collapse and hypoxemia in early acute respiratory distress syndrome. Am J Respir Crit Care Med. 2006;174:268-78. [PMID: 16690982] 


\section{Annals of Internal Medicine}

Current Author Addresses: Drs. Putensen, Theuerkauf, Zinserling, and Wrigge: Department of Anaesthesiology and Intensive Care Medicine, University of Bonn, Sigmund-Freud-Strasse 25, 53105 Bonn, Germany. Dr. Pelosi: Department of Ambient, Health, and Safety, University of Insubria, Servizio di Anestesia B, Ospedale di Circolo e Fondazione Macchi viale Borri 57, 21100 Varese, Italy.

Author Contributions: Conception and design: C. Putensen, P. Pelosi. Analysis and interpretation of the data: C. Putensen, N. Theuerkauf, J. Zinserling, H. Wrigge, P. Pelosi.
Drafting of the article: C. Putensen, P. Pelosi.

Critical revision of the article for important intellectual content: C. Putensen, P. Pelosi.

Final approval of the article: C. Putensen, P. Pelosi.

Provision of study materials or patients: C. Putensen.

Statistical expertise: J. Zinserling.

Obtaining of funding: C. Putensen.

Administrative, technical, or logistic support: C. Putensen.

Collection and assembly of data: C. Putensen, N. Theuerkauf, J. Zinserling, H. Wrigge, P. Pelosi. 
Appendix Table 1. Characteristics of the Study Participants, Therapies, and Outcomes

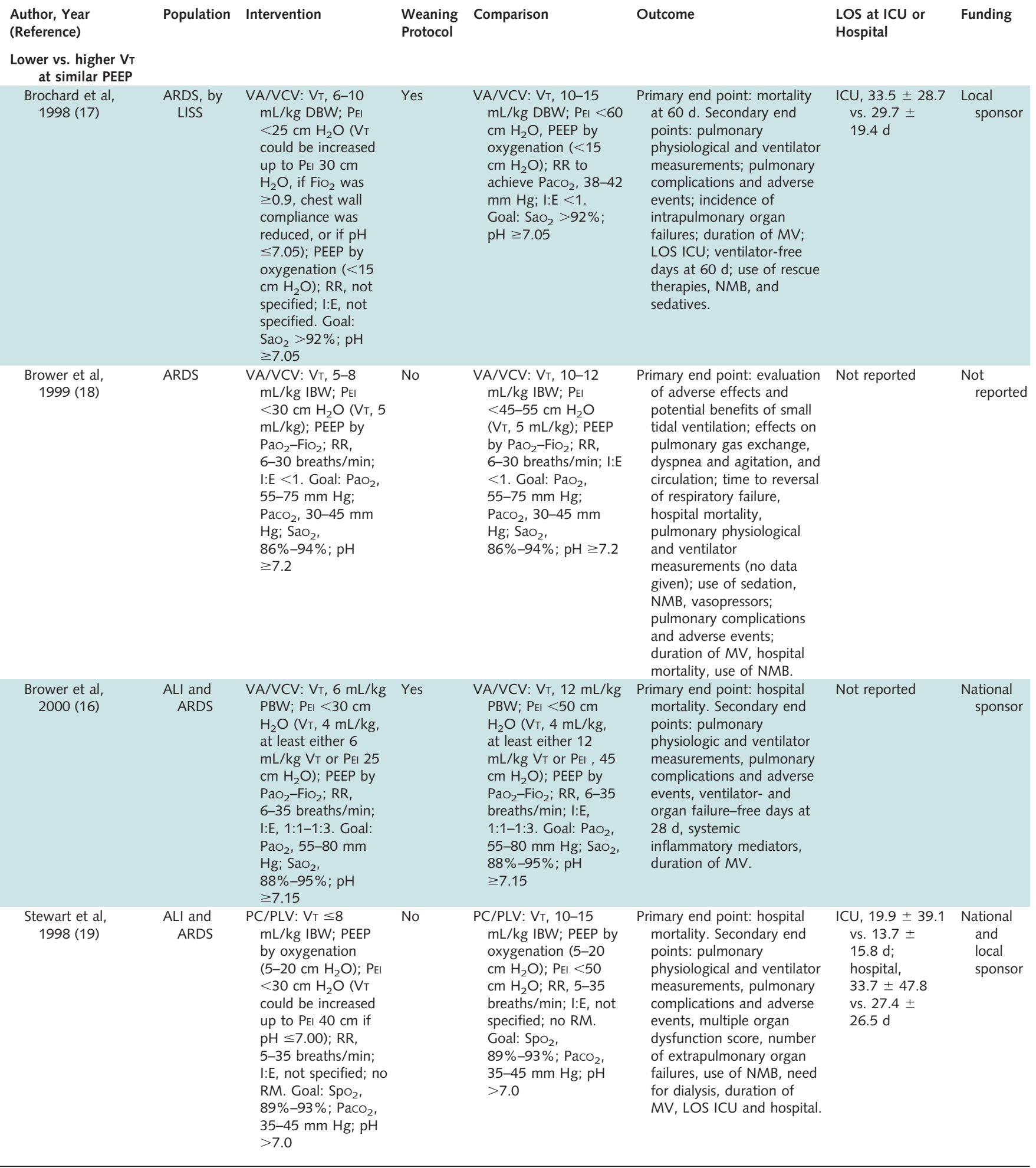




\begin{tabular}{|c|c|c|c|c|c|c|c|}
\hline $\begin{array}{l}\text { Author, Year } \\
\text { (Reference) }\end{array}$ & Population & Intervention & $\begin{array}{l}\text { Weaning } \\
\text { Protocol }\end{array}$ & Comparison & Outcome & $\begin{array}{l}\text { LOS at ICU or } \\
\text { Hospital }\end{array}$ & Funding \\
\hline \multicolumn{8}{|c|}{$\begin{array}{l}\text { Lower vs. higher PEEP } \\
\text { at low } \mathrm{V}_{T}\end{array}$} \\
\hline $\begin{array}{c}\text { Brower et al, } \\
2004(20)\end{array}$ & $\begin{array}{r}\text { ALI and } \\
\text { ARDS }\end{array}$ & $\begin{array}{l}\text { VA/VCV: } \mathrm{VT}, 6 \mathrm{~mL} / \mathrm{kg} \\
\mathrm{PBW} ; \mathrm{PEI}<30 \mathrm{~cm} \\
\mathrm{H}_{2} \mathrm{O} ; \text { high PEEP by } \\
\mathrm{PaO}_{2}-\mathrm{FiO}_{2} ; \mathrm{RR} \leq 35 \\
\text { breaths/min; I:E, } \\
\text { 1:1-1:3; RM in the } \\
\text { first } 80 \text { points. Goal: } \\
\mathrm{PaO}_{2}, 55-80 \mathrm{~mm} \\
\mathrm{Hg}^{\mathrm{SgaO}}, \\
88 \%-95 \% ; \mathrm{pH} \\
\geq 7.3\end{array}$ & Yes & $\begin{array}{l}\text { VA/VCV: VT, } 6 \mathrm{~mL} / \mathrm{kg} \\
\text { PBW; PEI <30 cm } \\
\mathrm{H}_{2} \mathrm{O} ; \mathrm{low} \text { PEEP by } \\
\mathrm{PaO}_{2}-\mathrm{FiO}_{2} ; \mathrm{RR} \leq 35 \\
\text { breaths/min; I:E, } \\
\text { 1:1-1:3; no RM. } \\
\text { Goal: } \mathrm{PaO}_{2}, 55-80 \\
\mathrm{~mm} \mathrm{Hg} \mathrm{SaO}_{2} ; \\
88 \%-95 \% ; \mathrm{pH} \geq 7.3\end{array}$ & $\begin{array}{l}\text { Primary end point: hospital } \\
\text { mortality. Secondary end } \\
\text { points: pulmonary } \\
\text { physiologic and ventilator } \\
\text { measurements, pulmonary } \\
\text { complications and adverse } \\
\text { events, ventilator- and } \\
\text { organ failure-free days at } \\
28 \text { d, systemic inflam- } \\
\text { matory mediators. }\end{array}$ & Not reported & $\begin{array}{l}\text { National } \\
\text { sponsor }\end{array}$ \\
\hline $\begin{array}{l}\text { Meade et al, } \\
2008(21)\end{array}$ & $\begin{array}{r}\text { ALI and } \\
\text { ARDS }\end{array}$ & $\begin{array}{l}\mathrm{PCV}: \mathrm{VT}, 6 \mathrm{~mL} / \mathrm{kg} \\
\mathrm{PBW} ; \mathrm{PEl}<40 \mathrm{~cm} \\
\mathrm{H}_{2} \mathrm{O} ; \text { high PEEP by } \\
\mathrm{PaO}_{2}-\mathrm{FiO}_{2} ; \mathrm{RR} \leq 35 \\
\text { breaths/min; I:E, } \\
\text { 1:1-1:3; recruitment } \\
\text { maneuver at start of } \\
\text { the study and after } \\
\text { each disconnect } \\
\text { from the ventilator. } \\
\text { Goal: } \mathrm{PaO}_{2}, 55-80 \\
\text { mm } \mathrm{Hg}_{2} \mathrm{SaO}_{2}, \\
88 \%-93 \% ; \mathrm{pH} \\
\geq 7.3\end{array}$ & Yes & $\begin{array}{l}\text { VA/VCV: } \mathrm{VT}_{\mathrm{T}}, 6 \mathrm{~mL} / \mathrm{kg} \\
\text { PBW, PEl }<30 \mathrm{~cm} \\
\mathrm{H}_{2} \mathrm{O}, \text { low PEEP by } \\
\mathrm{PaO}_{2}-\mathrm{FiO}_{2}, \mathrm{RR} \leq 35 \\
\text { I:E, 1:1-1:3; no RM. } \\
\text { Goal: } \mathrm{PaO}_{2}, 55-80 \\
\mathrm{~mm} \mathrm{Hg} \mathrm{SaO}_{2} \\
88 \%-93 \% ; \mathrm{pH} \geq 7.3\end{array}$ & $\begin{array}{l}\text { Primary end point: hospital } \\
\text { mortality, ICU mortality, } \\
\text { 28-d mortality, and } \\
\text { mortality rate during MV. } \\
\text { Secondary end points: } \\
\text { pulmonary physiologic and } \\
\text { ventilator measurements; } \\
\text { pulmonary complications } \\
\text { and adverse events; use of } \\
\text { co-interventions and rescue } \\
\text { therapies; duration of MV; } \\
\text { LOS ICU and hospital; } \\
\text { need for sedatives, } \\
\text { narcotics, NMB, dialysis, } \\
\text { vasopressors, and } \\
\text { corticosteroids. }\end{array}$ & $\begin{array}{l}\text { Median ICU, } 13 \\
\text { vs. } 13 \mathrm{~d} ; \\
\text { hospital, } 28 \text { vs. } \\
29 \mathrm{~d}\end{array}$ & $\begin{array}{l}\text { Local and } \\
\text { national } \\
\text { sponsor }\end{array}$ \\
\hline $\begin{array}{c}\text { Mercat et al, } \\
2008(22)\end{array}$ & $\begin{array}{r}\text { ALI and } \\
\text { ARDS }\end{array}$ & $\begin{array}{l}\text { VA/VCV: } \mathrm{VT}, 6 \mathrm{~mL} / \mathrm{kg} \\
\text { PBW; PEI }<30 \mathrm{~cm} \\
\mathrm{H}_{2} \mathrm{O} ; \mathrm{PEEP} \text { as high } \\
\text { as possible with PEI } \\
28-30 \mathrm{~cm} \mathrm{H}_{2} \mathrm{O} ; \mathrm{RR} \\
\leq 35 \text { breaths/min; } \\
\text { I:E, not specified; } \\
\text { recruitment } \\
\text { maneuver allowed } \\
\text { but not } \\
\text { recommended. } \\
\text { Goal: } \mathrm{PaO}_{2}, 55-80 \\
\text { mm } \mathrm{Hg}^{\circ} \mathrm{SaO}_{2}, \\
88 \%-95 \% ; \mathrm{pH}, \\
7.3-7.45\end{array}$ & Yes & $\begin{array}{l}\text { VA/VCV: } \mathrm{VT}, 6 \mathrm{~mL} / \mathrm{kg} \\
\text { PBW; PEI }<30 \mathrm{~cm} \\
\mathrm{H}_{2} \mathrm{O} ; \mathrm{PEEP} \text { as low as } \\
\text { possible, } 5-9 \mathrm{~cm} \\
\mathrm{H}_{2} \mathrm{O} ; \mathrm{RR} \leq 35 \\
\text { breaths/min; I:E, not } \\
\text { specified; recruitment } \\
\text { maneuver allowed } \\
\text { but not } \\
\text { recommended. Goal: } \\
\mathrm{PaO}_{2}, 55-80 \mathrm{~mm} \mathrm{Hg} \\
\mathrm{SaO}_{2}, 88 \%-95 \% ; \mathrm{pH} \\
\geq 7.3-7.45\end{array}$ & $\begin{array}{l}\text { Primary end point: mortality } \\
\text { at } 28 \mathrm{~d} \text {. Secondary end } \\
\text { points: hospital mortality; } \\
\text { pulmonary physiologic and } \\
\text { ventilator measurements; } \\
\text { pulmonary complications } \\
\text { and adverse events; } \\
\text { mortality at } 60 \mathrm{~d} \text {; } \\
\text { ventilator-free and organ } \\
\text { failure-free days at } 28 \mathrm{~d} ; \\
\text { use of co-interventions, } \\
\text { NMB, vasopressors, and } \\
\text { rescue therapies. }\end{array}$ & Not reported & $\begin{array}{l}\text { Local and } \\
\text { national } \\
\text { sponsor }\end{array}$ \\
\hline
\end{tabular}

Lower $V_{T}+$ higher

PEEP vs. higher $\mathrm{V}_{\mathrm{T}}+$ lower PEEP

Amato et al, 1998 (23)

\begin{tabular}{|c|c|}
\hline $\begin{array}{c}\text { ARDS by } \\
\text { LISS }\end{array}$ & $\begin{array}{l}\text { PC/PLV: } \mathrm{V}_{\mathrm{T}} \leq 6 \\
\mathrm{~mL} / \mathrm{kg} \mathrm{BW} ; \mathrm{PEEP}, \\
\mathrm{LIP}+2 \mathrm{~cm} \mathrm{H} \mathrm{H}_{2} \mathrm{O} \text { or } \\
16 \mathrm{~cm} \mathrm{H}_{2} \mathrm{O} ; \mathrm{PEI} \\
<\mathrm{PEEP}+20 ; \mathrm{RR} \\
<30 \text { breaths/min; } \\
\mathrm{I}: \mathrm{E}>1, \text { recruitment } \\
\text { maneuver } \\
\text { "frequently used," } \\
\text { especially after each } \\
\text { disconnect from the } \\
\text { ventilator. Goal: } \\
\text { PaO }, 80 \text { mm } \mathrm{Hg} ; \\
\text { permissive } \\
\text { hypercapnia, } \mathrm{pH} \\
>7.2\end{array}$ \\
\hline
\end{tabular}

\author{
VA/VCV: $\mathrm{VT}_{\mathrm{T}} 12 \mathrm{~mL} / \mathrm{kg}$ \\ BW; PEEP by \\ oxygenation $\geq 5 \mathrm{~cm}$ \\ $\mathrm{H}_{2} \mathrm{O} ; \mathrm{PEI}$, not limited; \\ RR, 10-24 \\ breaths/min or $\mathrm{PaCO}_{2}$ \\ $>25 \mathrm{~mm} \mathrm{Hg}$; I:E, not \\ specified; no \\ recruitment \\ maneuver. Goal: \\ $\mathrm{PaO}_{2}, 80 \mathrm{~mm} \mathrm{Hg}$; \\ $\mathrm{PaCO}_{2}, 35-38 \mathrm{~mm}$ \\ $\mathrm{Hg}$
}

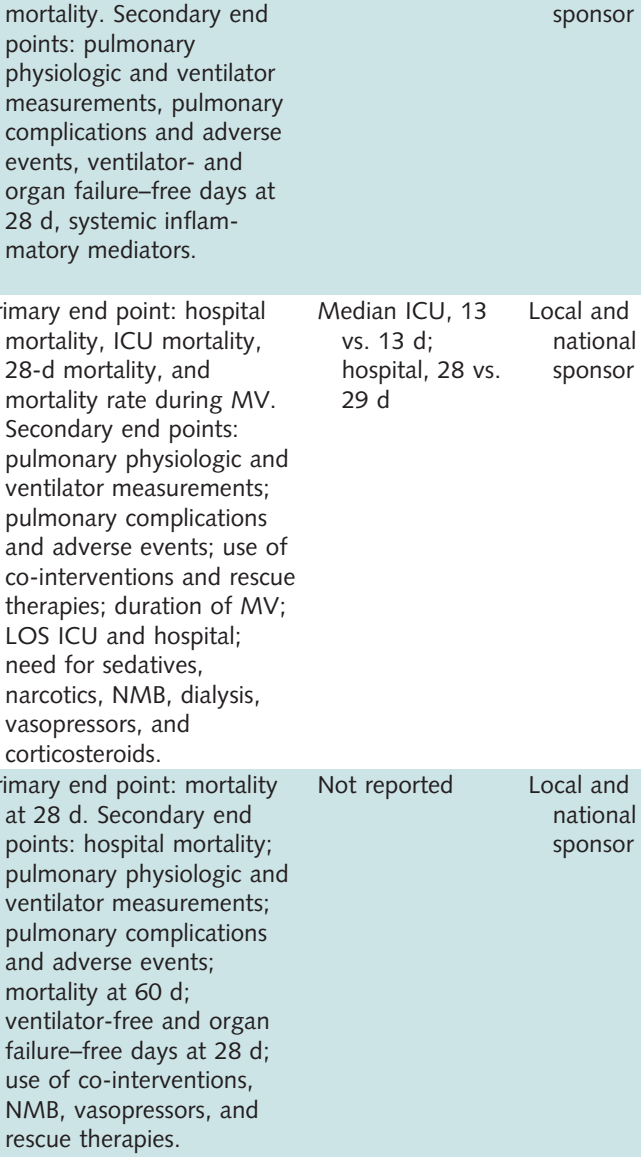
physiologic and ventilator measurements, pulmonary plications and adverse temic inflam-

imary end point: hospita mortality, and adverse events; use of co-interventions and rescue LOS ICU and hospital; need for sedatives, vasopressors, and corticosteroids. at $28 \mathrm{~d}$. Secondary end points: hospital mortality; pulmonary physiologic and ventilator measurements ( mortality at $60 \mathrm{~d}$; ventilator-free and organ failure-free days at $28 \mathrm{~d}$ of co-interventions, rescue therapies. sponsor sponsor

\section{OS at ICU or Funding}

Not reported Nationa 


\begin{tabular}{|c|c|c|c|c|c|c|c|}
\hline \multicolumn{8}{|c|}{ Appendix Table 1-Continued } \\
\hline $\begin{array}{l}\text { Author, Year } \\
\text { (Reference) }\end{array}$ & Population & Intervention & $\begin{array}{l}\text { Weaning } \\
\text { Protocol }\end{array}$ & Comparison & Outcome & $\begin{array}{l}\text { LOS at ICU or } \\
\text { Hospital }\end{array}$ & Funding \\
\hline $\begin{array}{l}\text { Villar et al, } \\
2006 \text { (24) }\end{array}$ & ARDS & $\begin{array}{l}\text { VA/VCV: VT, 5-8 } \\
\mathrm{mL} / \mathrm{kg} \mathrm{PBW} ; \mathrm{PEEP}, \\
\mathrm{LIP}+2 \mathrm{~cm} \mathrm{H}_{2} \mathrm{O} \text { or } \\
15 \mathrm{~cm} \mathrm{H}_{2} \mathrm{O} ; \mathrm{PEl} \text {, not } \\
\text { specified; RR by } \\
\text { Paco }_{2} \text { I: } \mathrm{E}, \text { not } \\
\text { specified; no RM. } \\
\text { Goal: } \mathrm{PaO}_{2}, 70-100 \\
\mathrm{~mm} \mathrm{Hg} \mathrm{SaO}_{2}>90 \% \text {; } \\
\mathrm{PacO}_{2}, 35-50 \mathrm{~cm} \mathrm{H} \mathrm{H}_{2} \mathrm{O}\end{array}$ & No & $\begin{array}{l}\text { VA/VCV: } V_{T}, 9-11 \\
\text { mL/kg PBW; PEEP by } \\
\text { oxygenation } \geq 5 \mathrm{~cm} \\
\mathrm{H}_{2} \mathrm{O} ; \text { PEl, not specified; } \\
\mathrm{RR} \text { by PaCO } ; \text { I:E, not } \\
\text { specified; no RM. Goal: } \\
\mathrm{PaO}_{2}, 70-100 \mathrm{~mm} \mathrm{Hg} ; \\
\mathrm{SaO}_{2}>90 \% ; \mathrm{PaCO}_{2}, \\
35-50 \mathrm{~cm} \mathrm{H} \mathrm{O}\end{array}$ & $\begin{array}{l}\text { Primary end point: ICU } \\
\text { mortality. Secondary end } \\
\text { points: hospital mortality, } \\
\text { pulmonary physiologic and } \\
\text { ventilator measurements, } \\
\text { pulmonary complications and } \\
\text { adverse events, ventilator- } \\
\text { free days at } 28 \mathrm{~d} \text {, number of } \\
\text { extrapulmonary organ } \\
\text { failures. }\end{array}$ & Not reported & $\begin{array}{l}\text { Nationa } \\
\text { sponsor }\end{array}$ \\
\hline
\end{tabular}

ALI $=$ acute lung injury; APACHE $=$ Acute Physiology and Chronic Health Evaluation; ARDS $=$ acute respiratory distress syndrome; BW $=$ body weight; DBW $=$ dry body weight (defined as actual body weight minus estimated weight gain due to water and salt retention); IBW = ideal body weight; ICU = intensive care unit; I:E = inspiratory:expiratory time; LIP = lower inflection point; LISS = Lung Injury Severity Score; LOS = length of stay; MV = mechanical ventilation; NMB = neuromuscular blockers; $\mathrm{PBW}=$ predicted body weight; $\mathrm{PC} / \mathrm{PLV}=$ pressure-control/pressure-limited ventilation; $\mathrm{PCV}=$ pressure-control ventilation; $\mathrm{PEEP}=$ positive end-expiratory pressure; $\mathrm{PEI}=$ end-inspiratory pressure; $\mathrm{RM}=$ recruitment maneuver; $\mathrm{RR}=$ respiratory rate; $\mathrm{VA} / \mathrm{VCV}=$ volume-assist/volume-control ventilation; $\mathrm{VT}=$ tidal volume. 


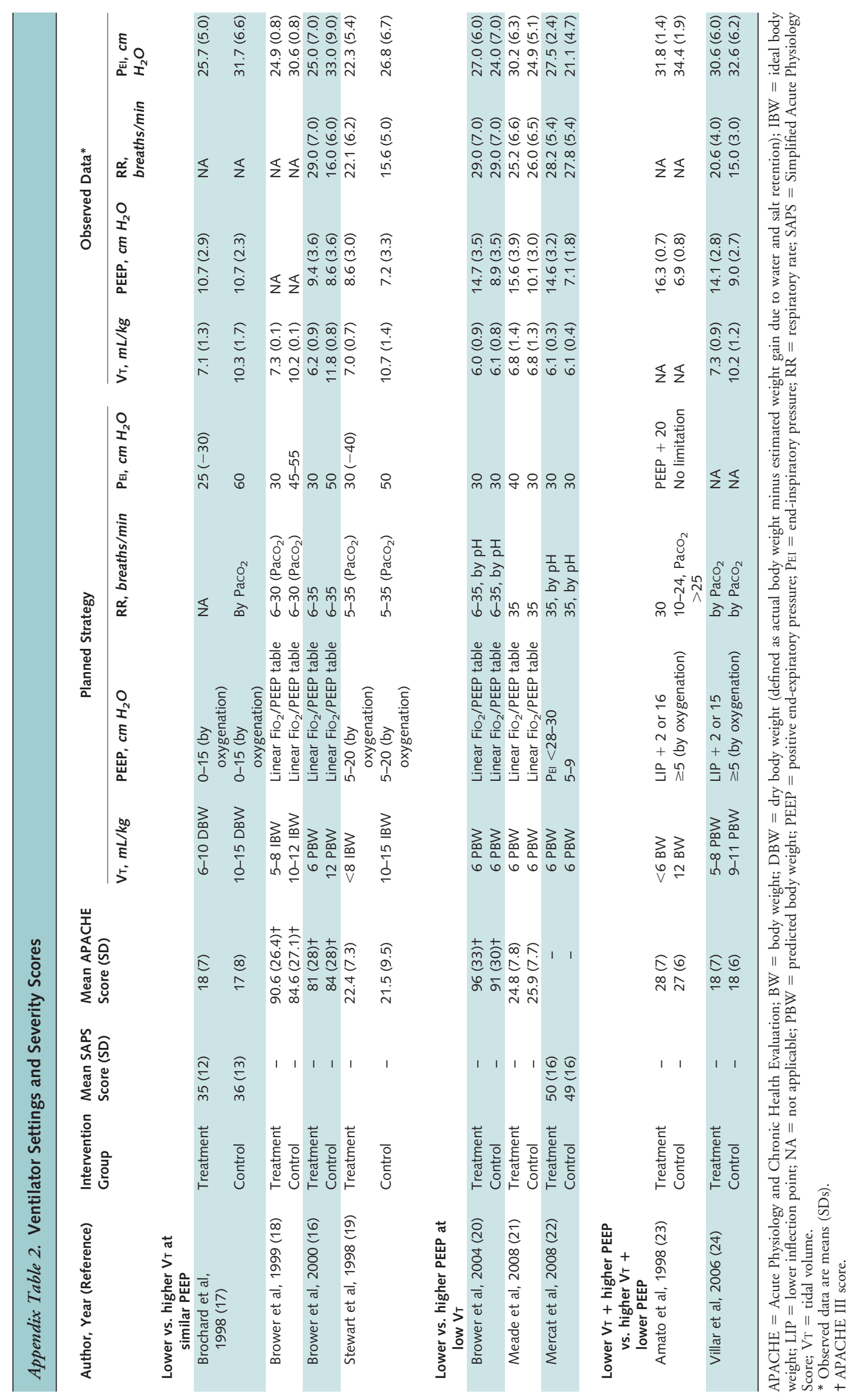




\section{Appendix Table 3. Management of pH}

\begin{tabular}{|c|c|c|c|}
\hline Author, Year (Reference) & Study Group & $\mathrm{pH}$ Threshold & Planned Intervention \\
\hline \multicolumn{4}{|c|}{ Lower vs. higher $\mathrm{V}_{\mathrm{T}}$ at similar PEEP } \\
\hline \multirow[t]{2}{*}{ Brochard et al, 1998 (17) } & Treatment & 7.05 & $\begin{array}{l}\text { If } \mathrm{pH}<7.05 \text {, increase } \mathrm{V}_{\mathrm{T}} \text { until } \mathrm{PEI} \max \text { is } 30 \mathrm{~cm} \mathrm{H} \mathrm{H}_{2} \mathrm{O} \text {; sodium } \\
\text { bicarbonate (not specified); dialysis for metabolic acidosis. }\end{array}$ \\
\hline & Control & Not specified & Not specified. \\
\hline \multirow[t]{2}{*}{ Brower et al, 1999 (18) } & Treatment & 7.30 & $\begin{array}{l}\text { If } \mathrm{pH}<7.3 \text {, sodium bicarbonate permissible. If } \mathrm{pH}<7.2 \text {, sodium } \\
\text { bicarbonate }(10 \mathrm{mEq} / \mathrm{h}) \text { required. }\end{array}$ \\
\hline & Control & 7.30 & $\begin{array}{l}\text { If } \mathrm{pH}<7.3 \text {, sodium bicarbonate permissible. If } \mathrm{pH}<7.2 \text {, sodium } \\
\text { bicarbonate }(10 \mathrm{mEq} / \mathrm{h}) \text { required. }\end{array}$ \\
\hline \multirow[t]{2}{*}{ Brower et al, 2000 (16) } & Treatment & 7.15 & If $\mathrm{pH}<7.15$ increase $\mathrm{PEI}$ up to $\geq 30 \mathrm{~cm} \mathrm{H}_{2} \mathrm{O}$. \\
\hline & Control & 7.15 & If $\mathrm{pH}<7.15$ increase $\mathrm{PEl}$ up to $\geq 50 \mathrm{~cm} \mathrm{H}_{2} \mathrm{O}$. \\
\hline \multirow[t]{2}{*}{ Stewart et al, 1998 (19) } & Treatment & 7.00 & $\begin{array}{l}\text { If } \mathrm{pH}<7.0,2 \mathrm{mmol} / \mathrm{kg} \text { sodium bicarbonate max } 3 \text { times per day, } \\
\text { increase PPEAK to max } 40 \mathrm{~cm} \mathrm{H}_{2} \mathrm{O} \text {. If refractory acidosis }<7.0 \text {, } \\
\text { withdrawal from study. }\end{array}$ \\
\hline & Control & 7.00 & If $\mathrm{pH}<7.0,2 \mathrm{mmol} / \mathrm{kg}$ sodium bicarbonate max 3 times per day. \\
\hline \multicolumn{4}{|c|}{ Lower vs. higher PEEP at low $\mathrm{V}_{\mathrm{T}}$} \\
\hline \multirow[t]{2}{*}{ Brower et al, 2004 (20) } & Treatment & 7.30 & $\begin{array}{l}\text { If } \mathrm{pH} 7.15 \text { to } 7.3 \text {, increase respiratory rate to max } 35 \text { breaths/min. } \\
\text { If respiratory rate is } 35 \text { breaths/min, give sodium bicarbonate. If } \\
\mathrm{pH}<7.15 \text {, increase respiratory rate to max } 35 \text { breaths/min. If } \\
\text { respiratory rate is } 35 \text { breaths } / \mathrm{min} \text { and sodium bicarbonate has } \\
\text { been given, increase } \mathrm{VT} \text { by } 1 \mathrm{~mL} / \mathrm{kg} \text { until } \mathrm{pH}>7.1 \text { (PEl may be } \\
\text { exceeded). }\end{array}$ \\
\hline & Control & 7.30 & $\begin{array}{l}\text { If } \mathrm{pH} 7.15 \text { to } 7.3 \text {, increase respiratory rate to } \max 35 \text { breaths/min. } \\
\text { If respiratory rate is } 35 \text { breaths/min, give sodium bicarbonate. If } \\
\mathrm{pH}<7.15 \text {, increase respiratory rate to max } 35 \text { breaths/min. If } \\
\text { respiratory rate is } 35 \text { breaths } / \mathrm{min} \text { and sodium bicarbonate has } \\
\text { been given, increase } \mathrm{VT} \text { by } 1 \mathrm{~mL} / \mathrm{kg} \text { until } \mathrm{pH}>7.1 \text { (PEl may be } \\
\text { exceeded). }\end{array}$ \\
\hline \multirow[t]{2}{*}{ Meade et al, 2008 (21) } & Treatment & $\begin{array}{l}<7.1 \text { for } \\
>1 \mathrm{~h}\end{array}$ & $\begin{array}{l}\text { Protocol deviation or rescue therapy, prone position, iNO, HFV, } \\
\text { ECMO. }\end{array}$ \\
\hline & Control & $\begin{array}{l}<7.1 \text { for } \\
>1 \mathrm{~h}\end{array}$ & $\begin{array}{l}\text { Protocol deviation or rescue therapy, prone position, iNO, HFV, } \\
\text { ECMO. }\end{array}$ \\
\hline \multirow[t]{2}{*}{ Mercat et al, 2008 (22) } & Treatment & 7.30 & $\begin{array}{l}\text { If } \mathrm{pH}<7.3 \text {, give sodium bicarbonate. If } \mathrm{pH}<7.15 \text {, increase } \mathrm{V}_{\mathrm{T}} \\
\text { until max } 8 \mathrm{~mL} / \mathrm{kg} \text { and } \mathrm{PEI}_{\mathrm{EI}} \max 32 \mathrm{~cm} \mathrm{H} \mathrm{H}_{2} \mathrm{O} \text {, reduce PEEP to min } \\
5 \mathrm{~cm} \mathrm{H} \mathrm{H}_{2} \mathrm{O} \text {, maintain } V_{T} \min 4 \mathrm{~mL} / \mathrm{kg} \text {. }\end{array}$ \\
\hline & Control & 7.30 & $\begin{array}{l}\text { If } \mathrm{pH}<7.3 \text {, give sodium bicarbonate. If } \mathrm{pH}<7.15 \text {, increase } \mathrm{V}_{T} \\
\text { until } \max 8 \mathrm{~mL} / \mathrm{kg} \text { and } \mathrm{P}_{\mathrm{EI}} \max 32 \mathrm{~cm} \mathrm{H} \mathrm{H}_{2} \mathrm{O} \text {, reduce PEEP to min } \\
5 \mathrm{~cm} \mathrm{H} \mathrm{H}_{2} \mathrm{O} \text {, maintain } V_{T} \min 4 \mathrm{~mL} / \mathrm{kg} \text {. }\end{array}$ \\
\hline
\end{tabular}

\section{Lower $\mathrm{V}_{\mathrm{T}}+$ higher PEEP vs. higher $\mathrm{V}_{\mathrm{T}}+$ lower} PEEP

Amato et al, 1998 (23)

Villar et al, 2006 (24)
Treatment $\quad 7.20$

Control

Treatment

Control
Not specified

Not specified

Not specified
If $\mathrm{pH}<7.2$, sodium bicarbonate $<50 \mathrm{mmoL} / \mathrm{h}$.

Not specified.

Management of $\mathrm{pH}$ up to clinician.

Management of $\mathrm{pH}$ up to clinician.

$\mathrm{ECMO}=$ extracorporeal membrane oxygenation; HFV $=$ high-frequency ventilation; $\mathrm{iNO}=$ inhaled nitric oxide; $\max =$ maximum; min $=$ minimum; PPEAK $=$ peak inspiratory pressure; PEEP = positive end-expiratory pressure; PEI $=$ end-inspiratory pressure; $\mathrm{VT}=$ tidal volume. 
Appendix Table 4. Effect of Ventilation Strategies on Pulmonary Function

\begin{tabular}{|c|c|c|c|c|c|c|}
\hline Strategy & $\begin{array}{l}\text { Studies, } \\
n\end{array}$ & $\begin{array}{l}\text { Patients in Each } \\
\text { Group, } n / n\end{array}$ & $\begin{array}{l}\text { Weighted Mean } \\
\text { Difference }(95 \% \mathrm{Cl}) *\end{array}$ & $P$ Value & $P^{2}$ Statistic & $P$ Value \\
\hline \multicolumn{7}{|l|}{$\mathrm{V}_{\mathrm{T}}$, day 1} \\
\hline Lower vs. higher $V_{T}$ at similar PEEP & 3 & $550 / 547$ & $-4.18(-5.83$ to -2.53$)$ & $<0.001$ & 98.6 & $<0.001$ \\
\hline Lower vs. higher PEEP at low $\mathrm{V}_{T}$ & 3 & $1136 / 1163$ & $-0.01(-0.06$ to 0.04$)$ & 0.66 & 0 & 0.43 \\
\hline Lower $\mathrm{V}_{\mathrm{T}}+$ higher PEEP vs. higher $\mathrm{V}_{\mathrm{T}}+$ lower PEEP & 1 & $50 / 45$ & $-2.90(-3.33$ to 2.47$)$ & $<0.001$ & NA & NA \\
\hline \multicolumn{7}{|l|}{ PEEP, day 1} \\
\hline Lower vs. higher $\mathrm{V}_{\mathrm{T}}$ at similar PEEP & 3 & $550 / 547$ & $0.71(0.07$ to 1.35$)$ & 0.03 & 45.8 & 0.160 \\
\hline Lower vs. higher PEEP at low $\mathrm{V}_{T}$ & 3 & $1136 / 1163$ & $6.28(4.91$ to 7.64$)$ & $<0.001$ & 96.3 & $<0.001$ \\
\hline Lower $\mathrm{V}_{\mathrm{T}}+$ higher PEEP vs. higher $\mathrm{V}_{\mathrm{T}}+$ lower PEEP & 2 & $79 / 69$ & 7.28 (3.07 to 11.50$)$ & $<0.001$ & 98.0 & $<0.001$ \\
\hline \multicolumn{7}{|l|}{ PEl, day 1} \\
\hline Lower vs. higher $V_{T}$ at similar PEEP & 3 & $550 / 547$ & $-6.33(-8.52$ to -4.14$)$ & $<0.001$ & 78.2 & 0.010 \\
\hline Lower vs. higher PEEP at low $\mathrm{V}_{T}$ & 3 & $1136 / 1163$ & 4.96 (3.26 to 6.66$)$ & $<0.001$ & 93.6 & $<0.001$ \\
\hline Lower $\mathrm{V}_{\mathrm{T}}+$ higher PEEP vs. higher $\mathrm{V}_{\mathrm{T}}+$ lower PEEP & 2 & $79 / 69$ & $-2.53(-3.38$ to -1.67$)$ & $<0.001$ & 0 & 0.65 \\
\hline \multicolumn{7}{|l|}{ Respiratory rate, day 1} \\
\hline Lower vs. higher $\mathrm{V}_{\mathrm{T}}$ at similar PEEP & 2 & $492 / 489$ & $9.82(3.45$ to 16.18$)$ & 0.003 & 97.0 & $<0.001$ \\
\hline Lower vs. higher PEEP at low $\mathrm{V}_{T}$ & 3 & $1136 / 1163$ & $-0.14(-0.91$ to 0.64$)$ & 0.73 & 55.2 & 0.110 \\
\hline Lower $\mathrm{V}_{\mathrm{T}}+$ higher PEEP vs. higher $\mathrm{V}_{\mathrm{T}}+$ lower PEEP & 1 & $50 / 45$ & 5.60 (4.19 to 7.01$)$ & $<0.001$ & NA & NA \\
\hline \multicolumn{7}{|l|}{$\mathrm{PaO}_{2}$, day 1} \\
\hline Lower vs. higher $V_{T}$ at similar PEEP & 2 & $490 / 487$ & $-1.05(-3.83$ to 1.72$)$ & 0.46 & 0 & 0.84 \\
\hline Lower vs. higher PEEP at low $\mathrm{V}_{T}$ & 3 & $1136 / 1163$ & 11.06 (4.50 to 17.62$)$ & $<0.001$ & 85.1 & 0.001 \\
\hline Lower $\mathrm{V}_{\mathrm{T}}+$ higher PEEP vs. higher $\mathrm{V}_{\mathrm{T}}+$ lower PEEP & - & - & - & - & - & - \\
\hline \multicolumn{7}{|l|}{$\mathrm{PaCO}_{2}$, day 1} \\
\hline Lower vs. higher $\mathrm{V}_{\mathrm{T}}$ at similar PEEP & 2 & $490 / 487$ & $11.43(-1.50$ to 24.36$)$ & 0.080 & 97.0 & $<0.001$ \\
\hline Lower vs. higher PEEP at low $\mathrm{V}_{T}$ & 3 & $1136 / 1163$ & $0.77(-0.06$ to 1.59$)$ & 0.070 & 0 & 0.66 \\
\hline Lower $\mathrm{V}_{\mathrm{T}}+$ higher PEEP vs. higher $\mathrm{V}_{\mathrm{T}}+$ lower PEEP & 2 & $79 / 69$ & $9.68(-15.60$ to 34.96$)$ & 0.45 & 99.2 & $<0.001$ \\
\hline \multicolumn{7}{|l|}{$\mathrm{pH}$, day 1} \\
\hline Lower vs. higher $V_{T}$ at similar PEEP & 1 & $432 / 429$ & $-0.03(-0.04$ to -0.02$)$ & $<0.001$ & NA & NA \\
\hline Lower vs. higher PEEP at low $\mathrm{V}_{\mathrm{T}}$ & 3 & $1136 / 1163$ & $-0.01(-0.03$ to 0.00$)$ & 0.020 & 52.7 & 0.120 \\
\hline Lower $\mathrm{V}_{\mathrm{T}}+$ higher PEEP vs. higher $\mathrm{V}_{\mathrm{T}}+$ lower PEEP & 2 & $79 / 69$ & $-0.09(-0.27$ to 0.09$)$ & 0.31 & 99.1 & $<0.001$ \\
\hline \multicolumn{7}{|l|}{$\mathrm{V}_{\mathrm{T}}$, day 7} \\
\hline Lower vs. higher $V_{T}$ at similar PEEP & 3 & $550 / 547$ & $-3.86(-5.09$ to 2.63$)$ & $<0.001$ & 97.2 & $<0.001$ \\
\hline Lower vs. higher PEEP at low $\mathrm{V}_{T}$ & 3 & $1136 / 1163$ & $-0.03(-0.49$ to 0.44$)$ & 0.90 & 94.9 & $<0.001$ \\
\hline Lower $\mathrm{V}_{\mathrm{T}}+$ higher PEEP vs. higher $\mathrm{V}_{\mathrm{T}}+$ lower PEEP & 1 & $50 / 45$ & $-2.80(-3.23$ to -2.37$)$ & $<0.001$ & NA & NA \\
\hline \multicolumn{7}{|l|}{ PEEP, day 7} \\
\hline Lower vs. higher $\mathrm{V}_{\mathrm{T}}$ at similar PEEP & 3 & $550 / 547$ & $0.49(-1.29$ to 2.28$)$ & 0.59 & 90.7 & $<0.001$ \\
\hline Lower vs. higher PEEP at low $\mathrm{V}_{\mathrm{T}}$ & 3 & $1136 / 1163$ & $3.14(1.94$ to 4.34$)$ & $<0.001$ & 92.6 & $<0.001$ \\
\hline Lower $\mathrm{V}_{\mathrm{T}}+$ higher PEEP vs. higher $\mathrm{V}_{\mathrm{T}}+$ lower PEEP & 1 & $50 / 45$ & $-0.10(-1.55$ to 1.35$)$ & 0.89 & NA & NA \\
\hline \multicolumn{7}{|l|}{ PEI, day 7} \\
\hline Lower vs. higher $V_{T}$ at similar PEEP & 3 & $550 / 547$ & $-8.77(-11.61$ to -5.92$)$ & $<0.001$ & 84.1 & 0.002 \\
\hline Lower vs. higher PEEP at low $\mathrm{V}_{\mathrm{T}}$ & 3 & $1136 / 1163$ & 2.35 (0.40 to 4.30$)$ & 0.02 & 92.6 & $<0.001$ \\
\hline Lower $\mathrm{V}_{\mathrm{T}}+$ higher PEEP vs. higher $\mathrm{V}_{\mathrm{T}}+$ lower PEEP & 1 & $50 / 45$ & $-6.70(-9.77$ to -3.63$)$ & $<0.001$ & NA & NA \\
\hline \multicolumn{7}{|l|}{ Respiratory rate, day 7} \\
\hline Lower vs. higher $\mathrm{V}_{\mathrm{T}}$ at similar PEEP & 2 & $492 / 489$ & 7.95 (3.74 to 12.16$)$ & $<0.001$ & 93.0 & $<0.001$ \\
\hline Lower vs. higher PEEP at low $\mathrm{V}_{T}$ & 3 & $1136 / 1163$ & $0.14(-1.55$ to 1.82$)$ & 0.87 & 87.5 & $<0.001$ \\
\hline Lower $\mathrm{V}_{\mathrm{T}}+$ higher PEEP vs. higher $\mathrm{V}_{\mathrm{T}}+$ lower PEEP & 1 & $50 / 45$ & $1.90(0.07$ to 3.73$)$ & 0.040 & NA & NA \\
\hline \multicolumn{7}{|l|}{$\mathrm{PaO}_{2}$, day 7} \\
\hline Lower vs. higher $V_{T}$ at similar PEEP & 2 & $490 / 487$ & $-1.79(-4.25$ to 0.67$)$ & 0.150 & 0 & 0.55 \\
\hline Lower vs. higher PEEP at low $\mathrm{V}_{T}$ & 3 & $1136 / 1163$ & $0.02(-1.67$ to 1.70$)$ & 0.98 & 0 & 0.45 \\
\hline Lower $\mathrm{V}_{\mathrm{T}}+$ higher PEEP vs. higher $\mathrm{V}_{\mathrm{T}}+$ lower PEEP & - & - & - & - & - & - \\
\hline \multicolumn{7}{|l|}{$\mathrm{PaCO}_{2}$, day 7} \\
\hline Lower vs. higher $V_{T}$ at similar PEEP & 2 & $490 / 487$ & $9.88(-2.35$ to 22.11$)$ & 0.110 & 93.4 & 0.001 \\
\hline Lower vs. higher PEEP at low $\mathrm{V}_{T}$ & 3 & $1136 / 1163$ & $-1.37(-2.31$ to -0.43$)$ & 0.004 & 0 & 0.71 \\
\hline Lower $\mathrm{V}_{\mathrm{T}}+$ higher PEEP vs. higher $\mathrm{V}_{\mathrm{T}}+$ lower PEEP & 1 & $50 / 45$ & $-6.10(-10.75$ to -1.45$)$ & 0.010 & NA & NA \\
\hline \multicolumn{7}{|l|}{$\mathrm{pH}$, day 7} \\
\hline Lower vs. higher $\mathrm{V}_{\mathrm{T}}$ at similar PEEP & 1 & $432 / 429$ & $-0.01(-0.02$ to 0.00$)$ & 0.050 & NA & NA \\
\hline Lower vs. higher PEEP at low $\mathrm{V}_{\mathrm{T}}$ & 3 & $1136 / 1163$ & $0.00(0.00$ to 0.01$)$ & 0.17 & 7.9 & 0.34 \\
\hline Lower $\mathrm{V}_{\mathrm{T}}+$ higher PEEP vs. higher $\mathrm{V}_{\mathrm{T}}+$ lower PEEP & 1 & $50 / 45$ & 0.04 (0.01 to 0.07$)$ & 0.003 & NA & NA \\
\hline
\end{tabular}

$\mathrm{NA}=$ not applicable; PEEP $=$ positive end-expiratory pressure; $\mathrm{PEI}=$ end-inspiratory pressure; $\mathrm{VT}=$ tidal volume.

* Random-effects model.

$\mathbf{W - 1 9 0} \mid 20$ October 2009 $\mid$ Annals of Internal Medicine $\mid$ Volume $151 \cdot$ Number 8 


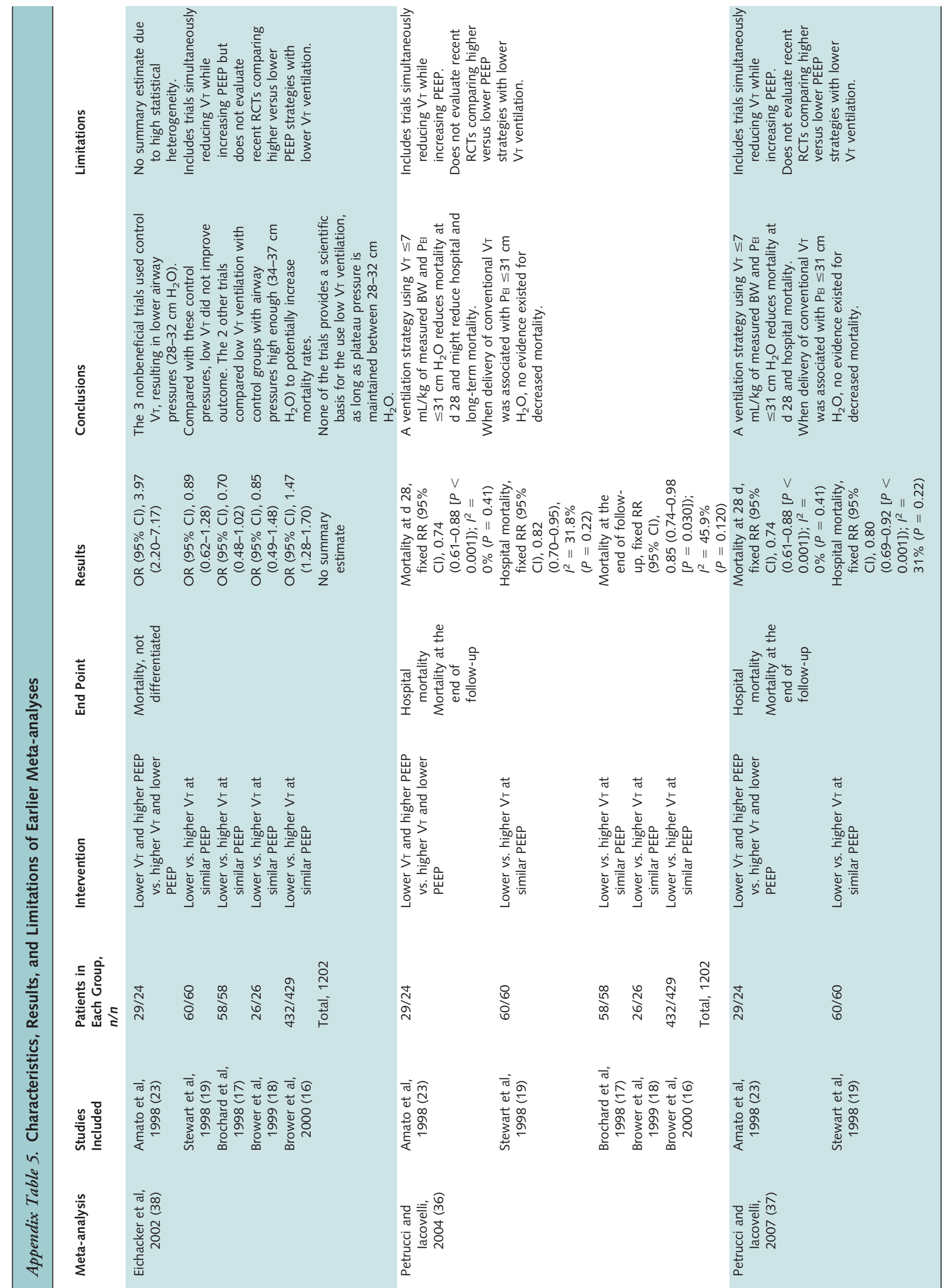




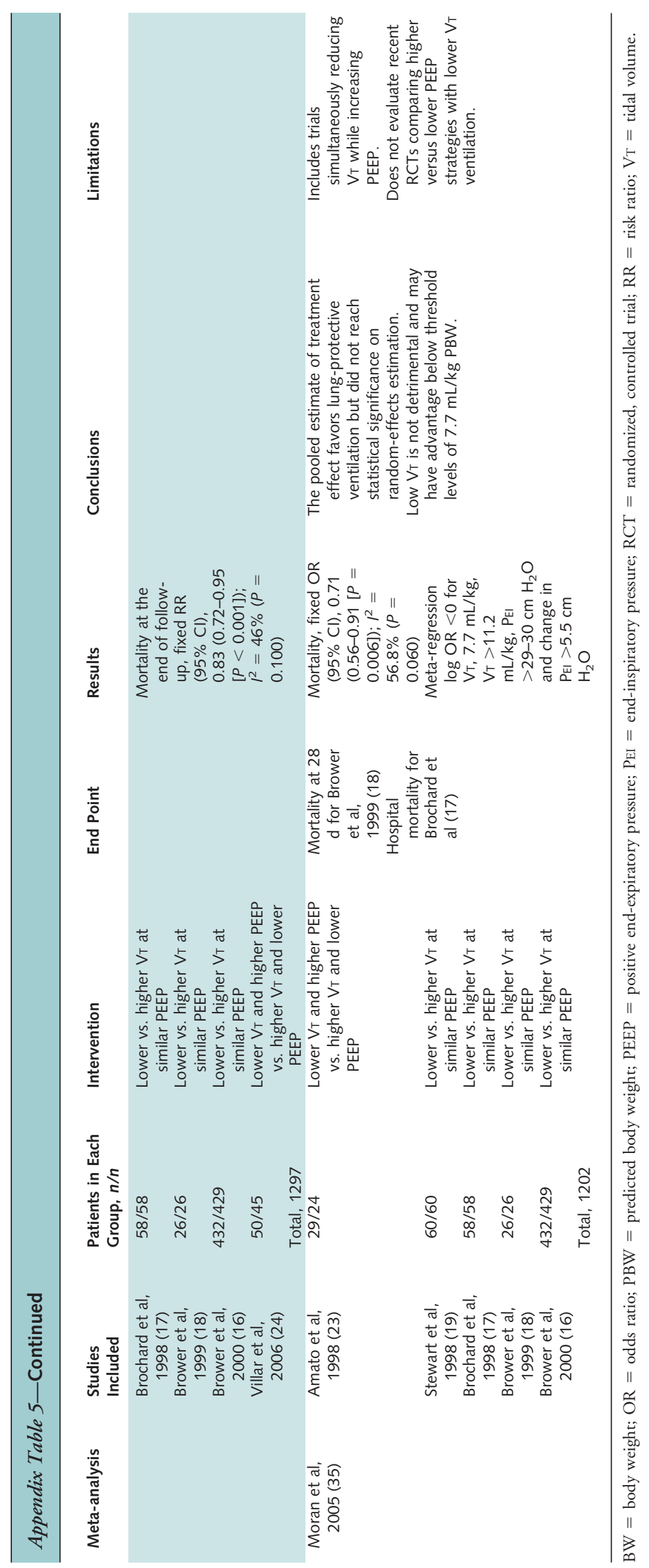

W-192 20 October 2009 $\mid$ Annals of Internal Medicine $\mid$ Volume 151 • Number 8 
Copyright of Annals of Internal Medicine is the property of American College of Physicians and its content may not be copied or emailed to multiple sites or posted to a listserv without the copyright holder's express written permission. However, users may print, download, or email articles for individual use. 